\title{
JUSTIFICATION OF MULTIDIMENSIONAL SINGLE PHASE SEMILINEAR GEOMETRIC OPTICS
}

\author{
JEAN-LUC JOLY AND JEFFREY RAUCH
}

ABstract. For semilinear strictly hyperbolic systems $L u=f(x, u)$, we construct and justify high frequency nonlinear asymptotic expansions of the form

$$
u^{\varepsilon}(x) \sim \sum_{j \geq 0} \varepsilon^{j} U_{j}(x, \varphi(x) / \varepsilon), \quad L u^{\varepsilon}-f\left(x, u^{\varepsilon}\right) \sim 0 .
$$

The study of the principal term of such expansions is called nonlinear geometric optics in the applied literature. We show

(i) formal expansions with periodic profiles $U_{j}$ can be computed to all orders,

(ii) the equations for the profiles from (i) are solvable, and

(iii) there are solutions of the exact equations which have the formal series as high frequency asymptotic expansion.

\section{INTRODUCTION}

Formal asymptotic solutions are one of the most useful tools of applied mathematicians. For linear partial differential equations some of the formal expansions have been molded into powerful tools which have transformed the subject in the last twenty years. This paper is devoted to a rigorous justification of some high frequency nonlinear asymptotic expansions. The study of the principal term of the expansion is well-known in the applied literature and goes under the name nonlinear geometric optics. The use of such expansions has been widespread for quasilinear hyperbolic systems [HK, MaRo, HMR, DM]. In this paper we will show that for semilinear hyperbolic systems in several variables and solutions with one phase, called oscillatory simple waves,

(i) the formal expansions can be computed to all orders, and

(ii) there are solutions of the exact equations which have the formal series as high frequency asymptotic expansion.

Point (ii), is the main result. The analogous questions in the quasilinear context have been studied by $O$. Gues [G] whose results are qualitatively similar.

Suppose that $x=\left(x_{0}, x_{1}, \ldots, x_{n}\right), \partial=\left(\partial_{0}, \ldots, \partial_{n}\right)$ and

$$
L(x, \partial)=\sum A_{j}(x) \partial_{j}+C(x),
$$

Received by the editors January 4, 1990.

1980 Mathematics Subject Classification (1985 Revision). Primary 35C20, 35L60.

Key words and phrases. Semilinear geometric optics, semilinear oscillating waves, high frequency asymptotic expansions.

Research of both authors was partically supported by NATO Grant CRG 890904.

Resarch of the second author was partially supported by NSF Grant DMS 8601783. 
is a strictly hyperbolic $N \times N$ system with smooth matrix valued coefficients and $x_{0}$ timelike. The objects of study are solutions, formal or exact, to the semilinear system

$$
L u=f(x, u)+b(x) .
$$

The asymptotic solutions of interest are of the form

$$
u^{\varepsilon}(x) \sim \sum_{j \geq 0} \varepsilon^{j} U_{j}(x, \varphi(x) / \varepsilon),
$$

solutions of

$$
L u^{\varepsilon}-f\left(x, u^{\varepsilon}\right) \sim \sum_{j \geq 0} \varepsilon^{j} B_{j}(x, \varphi(x) / \varepsilon) .
$$

Here $\varphi$ satisfies the eikonal equation $\operatorname{det}\left(L_{1}(x, d \varphi(x))=0\right.$ where $L_{1}$ denotes the principal symbol of $L$. The solutions represent high frequency waves oscillating transverse to the level sets of $\varphi$.

The choice of the amplitude, $0\left(\varepsilon^{0}\right)$ as $\varepsilon \rightarrow 0$, of $u^{\varepsilon}$ is crucial. Smaller solutions yield essentially linear perturbation theory and larger solutions have shrinking domain of existence as $\varepsilon \rightarrow 0$ (see [JR3] for more detail).

The interaction of two oscillatory simple waves for $2 \times 2$ systems admit similar asymptotic analysis and is discussed in $\S 8$. The $2 \times 2$ hypothesis eliminates the possibility of new resonant phases. The interaction of three waves can be radically more complicated [HMR, JR4] because of the possible creation of an infinity of new phases. This problem is not as severe in one space dimension [MaRo]. A justification of resonant expansions in dimension one is begun in [JR 1, 2] and completed, even in the quasilinear case before shock formation, in [JMR].

\section{FORMAL ASYMPTOTIC SOLUTIONS}

This section begins the study of formal asymptotic solutions (1.2), (1.3). The profiles $U_{j}(x, \theta)$ are smooth and $2 \pi$ periodic in $\theta$. One plugs (1.2) into the equation (1.3) to derive a sequence of nonlinear integro-differential equations for the profiles.

In $\S 3$ the unique solvability of these equations given appropriate initial data is proved. Roughly speaking the equations are no harder to solve than the Cauchy problem for $L$. On the other hand they are no easier to solve, so, pseudodifferential symmetrizers are needed in the strictly hyperbolic case.

The crucial third step, which is the key point of this paper, is to show that there are solutions of the associated Cauchy problem for $L$ to which the formal solutions are asymptotic. This is more difficult and is addressed in $\S \S 4$ and 5 .

For the first step one plugs in and expands $f\left(U_{0}(x, \varphi / \varepsilon)+\cdots\right)$ in a Taylor series about $U_{0}(x, \varphi / \varepsilon)$ with leading term $f\left(U_{0}(x, \varphi / \varepsilon)\right)$. Setting the coefficients of the powers $\varepsilon^{j}$ equal to zero for $k=-1,0$ and $k \geq 1$ yields the equations

$$
\begin{gathered}
\left(\sum A_{j} \partial_{j} \varphi\right) \partial_{\theta} U_{0}=0 \text { from } \varepsilon^{-1} \\
L\left(x, \partial_{x}\right) U_{0}-f\left(U_{0}\right)-B_{0}+\left(\sum A_{j} \partial_{j} \varphi\right) \partial_{\theta} U_{1}=0 \text { from } \varepsilon^{0} .
\end{gathered}
$$




$$
\begin{aligned}
& L\left(x, \partial_{x}\right) U_{k}-D_{u} f\left(x, U_{0}\right) U_{k}-B_{k}+\left(\sum A_{j} \partial_{j} \varphi\right) \partial_{\theta} U_{k+1} \\
& \quad+F_{k}\left(x, U_{0}, \ldots, U_{k-1}, B_{0}, \ldots, B_{k-1}\right)=0 \text { from } \varepsilon^{k}, \quad k \geq 1 .
\end{aligned}
$$

Equations (2.1) to (2.3) are to hold for all values of $x$ and $\theta$.

In order to have a solution $U_{0}$ with nontrivial $\theta$ dependence, which corresponds to rapid oscillation when $\varepsilon$ tends to zero, equation (2.1) forces

$$
\begin{gathered}
\operatorname{det}\left(\sum A_{j} \partial_{j} \varphi\right)=0, \quad \text { and } \\
\partial_{\theta} U_{0} \in \operatorname{ker}\left(\sum A_{j} \partial_{j} \varphi\right) .
\end{gathered}
$$

Equation (2.4) is the eikonal equation. It asserts that $d \varphi$ belongs to the characteristic variety of $L$. In the applied literature this is equivalent to the statement that $d \varphi(\underline{x})$ satisfies the high frequency dispersion relation of the operator $L(\underline{x}, \partial)$. Equation $(2.5)$ expresses a polarization. Only certain components of $u^{\varepsilon}$ are highly oscillatory.

The eikonal equation has solutions uniquely determined on a neighborhood of $\underline{x}$ with $t(\underline{x})=0$ by values of $\left.\varphi\right|_{t=0}$ satisfying $d \varphi(\underline{x}) \neq 0$. Fix such a solution $\varphi$. Since the set $t=0$ is noncharacteristic and the level sets of $\varphi$ are characteristic one can introduce new local coordinates, still denoted $x$, such that $x_{0}$ is preserved and $\varphi(x) \equiv x_{n}$. After multiplication by $A_{0}^{-1}$ in the new coordinates, $L$ is still given by (1.0) with $A_{0}=I$. The matrix $A_{n}$ has one-dimensional kernel since the planes $x_{n}=$ constant are simply characteristic [RR2]. A smoothly varying linear change of variable in $\mathbb{C}^{N}$ leaves $A_{0}=I$ and reduces $A_{n}$ to the block form

$$
A_{n}=\left[\begin{array}{cc}
0 & 0 \\
0 & \widetilde{A}_{n}
\end{array}\right]
$$

where $\widetilde{A_{n}}$ is a smooth invertible $(N-1) \times(N-1)$ matrix.

Introduce the notation [.] for the last $N-1$ components of an $N$ vector. The polarization expressed in $(2.5)$ is equivalent to $\partial_{\theta}\left[U_{0}\right]=0$. Equivalently,

$$
U_{0}(x, \theta)=\left(U_{0}^{1}(x, \theta), U_{0}^{2}(x), \ldots, U_{0}^{N}(x)\right) \equiv\left(U_{0}^{1}(x, \theta),\left[U_{0}\right](x)\right) .
$$

Let $E$ denote the operator which averages in the $\theta$ variable

$$
(E w)(x) \equiv \int_{0}^{2 \pi} w(x, \theta) d \theta / 2 \pi
$$

Let $\mathscr{E}$ denote the operator on $N$ tuples of functions of $x, \theta$ defined by

$$
\mathscr{E} \equiv \operatorname{diag}(I, E, \ldots, E) .
$$

Thus $\mathscr{E}$ leaves the first component unchanged and averages the others. The polarization identity $(2.5)$ has the following equivalent description:

$$
U_{0} \in \operatorname{ker}(I-\mathscr{E})=\operatorname{Rg}(\mathscr{E})
$$

In order to complete the determination of $U_{0}$ one must use equation (2.2) which involves both $U_{1}$ and $U_{0}$. The key observation, and this is typical in geometric optics, is that the operator on $U_{1}$ is not surjective. A condition on $U_{0}$ is obtained by requiring that $L\left(\partial_{x}\right) U_{0}-f\left(U_{0}\right)-B_{0}$ belongs to the range of 
$A_{n} \partial_{\theta}$. Thanks to the block form (2.6) this range consists exactly of functions whose first component is zero and whose last $N-1$ components have mean zero. This is precisely $\operatorname{ker}(\mathscr{E})$. Thus for $U_{0}$ we have the system

$$
\begin{gathered}
(I-\mathscr{E}) U_{0}=0, \quad \text { and } \\
\mathscr{E}\left(L\left(x, \partial_{x}\right) U_{0}-f\left(x, U_{0}\right)-B_{0}\right)=0 .
\end{gathered}
$$

Given $U_{0}$ satisfying these equations one obtains from (2.2) a formula for $\partial_{\theta}\left[U_{1}\right]$, namely

$$
\partial_{\theta}\left[U_{1}\right]=\widetilde{A}_{n}^{-1}\left[L U_{0}-f\left(x, U_{0}\right)-B_{0}\right] .
$$

Equation (2.11) asserts that the right-hand side has mean zero so it has a periodic primitive in $\theta$. Denote by $\partial_{\theta}^{-1}$ the operator which chooses the primitive which itself has mean zero. Let

$$
l_{1} \equiv l_{1}\left(x, U_{0}, B_{0}\right) \equiv\left(0, \partial_{\theta}^{-1}\left(\tilde{A}_{n}^{-1}\left[L U_{0}+f\left(U_{0}\right)-B_{0}\right]\right)\right) .
$$

Write $U_{1}=V_{1}+l_{1}$ defining $V_{1}$. Then $\partial_{\theta} V_{1}=0$ so $V_{1} \in \operatorname{ker}(I-\mathscr{E})$. In particular $(I-\mathscr{E}) U_{1}=(I-\mathscr{E}) l_{1}$ is determined.

The decomposition $U_{1}=V_{1}+l_{1}$ reveals two distinct sorts of oscillation in $U_{1}$. The term $l_{1}$ describes oscillations in the last $N-1$ components, which are inherited from the principal term $U_{0}$. The term $V_{1}$ describes oscillations in the first component, that is with the same polarization as $U_{0}$.

To complete the determination of $U_{1}$ equation (2.3) for $k=1$ is used. By analogy with $(2.10),(2.11)$ the $\mathscr{E}$ projection of this equation is sufficient to determine $U_{1}$. Thus the system for $U_{1}$ is

$$
\begin{aligned}
& (I-\mathscr{E}) U_{1}=l_{1}\left(x, U_{0}, B_{0}\right), \\
& \mathscr{E}\left(L\left(x, \partial_{x}\right) U_{1}-D_{u} f\left(U_{0}\right) U_{1}-B_{1}\right)=0 .
\end{aligned}
$$

In the same fashion equation (2.3) for $k-1$ and $k$ with $k \geq 1$ yields

$$
\begin{gathered}
(I-\mathscr{E}) U_{k}=l_{k}\left(x, U_{0}, \ldots, U_{k-1}, B_{0}, \ldots, B_{k-1}\right), \\
\mathscr{E}\left\{L\left(x, \partial_{x}\right) U_{k}-D_{u} f\left(x, U_{0}\right) U_{k}-B_{k}-F_{k}\left(x, U_{0}, \ldots, U_{k-1}\right)\right\}=0,
\end{gathered}
$$

where

$$
l_{k}=\left(0, \partial_{\theta}^{-1}\left(\tilde{A}_{n}^{-1}\left[L U_{k-1}+D_{u} f\left(x, U_{0}\right) U_{k-1}-B_{k-1}-F_{k}\right]\right)\right) .
$$

These calculations prove the following proposition.

Proposition 2.1. Suppose that $\Omega$ is an open subset of $\mathbb{R}^{n+1}$ and $\varphi \in C^{\infty}(\Omega: \mathbb{R})$ satisfies (2.4) and $d \varphi(x) \neq 0$ for all $x \in \Omega$. The expansion (1.2) is a formal solution to (1.3) if and only if (2.10), (2.11) and (2.12), (2.13) for all $k \geq 1$ are satisfied in $\Omega \times S_{\theta}^{1}$.

There is a version of this calculation for finite sums

$$
u^{\varepsilon, M}(x) \equiv \sum_{j=0}^{M} \varepsilon^{j} U_{j}(x, \varphi(x) / \varepsilon) .
$$

Proposition 2.2. Suppose that $\Omega$ is an open subset of $\mathbb{R}^{n+1}, \varphi \in C^{\infty}(\Omega: \mathbb{R})$ satisfies the eikonal equation (2.4), $d \varphi(x) \neq 0$ for all $x \in \Omega$, and $u^{\varepsilon, M}$ is given by (2.14) with profiles $U_{k} \in C^{\infty}\left(\Omega \times S^{1}\right)$. Then, the following are equivalent: 
(i) $L u^{\varepsilon, M}-f\left(x, u^{\varepsilon, M}\right)-\sum_{j=0}^{M} \varepsilon^{j} B_{j}(x, \varphi(x) / \varepsilon)=\varepsilon^{M} H(x, \varphi(x) / \varepsilon)+$ $\varepsilon^{M+1} G(\varepsilon, x, \varphi(x) / \varepsilon)$, where $H(x, \theta), G(\varepsilon, x, \theta)$ are smooth and periodic in $\theta$ and $\mathscr{E} H=0$.

(ii) $U_{0}$ satisfies equations $(2.10),(2.11)$, and, for $k=1, \ldots, M ; U_{k}$ satisfies (2.12) and (2.13).

Example. The most interesting case is when $M=0$ in which case $U_{0}$ satisfies (2.10), (2.11) and the residual in part (ii) is of the form $H(x, \varphi / \varepsilon)$ with $\mathscr{E} H=$ 0 .

\section{SOlVABILITY OF THE EQUATIONS FOR THE PROFILES}

Equation (2.10) shows that $U_{0}$ is determined once $\mathscr{E} U_{0}$ is known. Equation (2.11) is a semilinear equation. We will show that it is uniquely solvable on a neighborhood of $\underline{x}$ with $t(\underline{x})=0$ when the initial values of $U_{0}$ on $\{t=0\} \times S^{1}$ are given. For $k \geq 1$ the value of $(I-\mathscr{E}) U_{k}$ is determined by $U_{0}, \ldots, U_{k-1}$. We will show that equations $(2.12)-(2.13)$, which are linear in $U_{k}$, are globally solvable, that is determine $U_{k}$ from its Cauchy data on domains where $U_{0}$ is known. Thus the restriction on the domain of solvability comes from two sources, the local solvability of the eikonal equation and the local solvability of (2.11). One obtains profiles $U_{k}$ defined on a neighborhood independent of $k$.

Since the construction of $U_{0}$ is a local problem in a neighborhood of $\underline{x}$, the data of the problem may be changed outside a small neighborhood of $\underline{x}$. Thus one can suppose that

$L(x, \partial)$ has constant coefficients for $|x| \geq r>0$, is:

(3.1) strictly hyperbolic, $A_{0}=I$, and $A_{n}$ is given by (2.6) with $\tilde{A}_{n}$ invertible.

(3.2) $f(x, U) \equiv 0$ for $|x| \geq r>0$.

(3.3) $\varphi=x_{n}$ satisfies the eikonal equation for all $x$.

The semilinear equation (2.11) is solved by Picard iteration. The crucial ingredient is a linear existence theorem. This same theorem directly solves (2.13).

Proposition 3.1. Suppose that $s \in \mathbb{R}, T>0,(3.1),(3.2)$, (3.3) are satisfied, and that $B \in L^{1}\left([0, T]: H^{s}\left(\mathbb{R}^{n} \times S^{1}\right)\right)$ and $\alpha \in H^{s}\left(\mathbb{R}^{n} \times S^{1}\right)$ satisfy $\mathscr{E} B=B$ and $\mathscr{E} \alpha=\alpha$. Then there is a unique $V \in C\left([0, T]: H^{s}\left(\mathbb{R}^{n} \times S^{1}\right)\right)$ satisfying.

$$
\begin{aligned}
& (I-\mathscr{E}) V=0, \\
& \mathscr{E} L\left(x, \partial_{x}\right) V=B, \quad \text { and } \\
& \left.V\right|_{t=0}=\alpha .
\end{aligned}
$$

In addition, for $0 \leq t \leq T, V$ satisfies

$$
\|V(t)\|_{H^{s}\left(\mathbb{R}^{n} \times S^{1}\right)} \leq c(s, T)\left(\|V(0)\|_{H^{s}\left(\mathbb{R}^{n} \times S^{1}\right)}+\int_{0}^{t}\|B(\sigma)\|_{H^{s}\left(\mathbb{R}^{n} \times S^{1}\right)} d \sigma\right) .
$$

The one dimensional case, $n=1$, is simpler than the case $n>1$. The reason is that in that case our special choice of coordinates quarantees that $\left[\mathscr{E}, \partial_{t}\right]=\left[\mathscr{E}, A_{1} \partial_{x}\right]=0$ so that $\mathscr{E}$ commutes with the principal part of $L$. For $n>1$ one always has $\left[\mathscr{E}, \partial_{j}\right]=0$ for all $j$ but generically $\left[\mathscr{E}, A_{j}\right] \neq 0$ for $j>1$ so that $[\mathscr{E}, L]$ is an operator of first order. 
Proof of Proposition 3.1. Let $\tilde{x} \equiv\left(x_{1}, \ldots, x_{n}\right), \tilde{\partial} \equiv\left(\partial_{1}, \ldots, \partial_{n}\right), \quad \widetilde{D} \equiv$ $i^{-1} \tilde{\partial}$, and, $A=A(x, \tilde{\partial}) \equiv \sum_{j=1}^{n} A_{j}(x) \partial_{j}$. Choose a symmetrizer $R(x, \widetilde{D}) \in$ $C^{\infty}\left([0, T]:\right.$ Op $\left.S^{0}\left(\mathbb{R}^{n} \times \mathbb{R}^{n}\right)\right)$ for the operator $L$, that is

$$
\begin{aligned}
& R(t)=R(t)^{*} \quad\left({ }^{*} \equiv \text { adjoint w.r.t. } L^{2}\left(\mathbb{R}^{n}\right) \text { scalar product }\right), \\
& R(t) \geq c I>0 \quad \text { for all } t \in \mathbb{R}, \\
& \operatorname{Re}(R A) \equiv\left(R A+(R A)^{*}\right) / 2 \in C^{\infty}\left([0, T]: \text { Op } S^{0}\left(\mathbb{R}^{n} \times \mathbb{R}^{n}\right)\right) .
\end{aligned}
$$

The usual choice for the principal symbol of $R$ is $\sum \pi_{j}(x, \xi)^{*} \pi_{j}(x, \xi)$ where $\pi_{j}$ are the spectral projections of $A(x, \xi)[T$, p. 77].

Let $(\cdot, \cdot)$ denote the scalar product in $L^{2}\left(\mathbb{R}^{n} \times S^{1}\right)$ and $\|\cdot\|$ the corresponding norm. The basic energy estimate (3.7) for $V \in H^{1}\left([0, T] \times \mathbb{R}^{n} \times S^{1}\right)$ is derived as follows:

$$
\frac{d}{d t}(R(t) V(t), V(t))=\left(R_{t} V, V\right)+\left(R V_{t}, V\right)+\left(R V, V_{t}\right) .
$$

Thanks to the selfadjointness of $R$ the last two terms sum to twice the real part of $\left(R V_{t}, V\right)$. Note that $V_{t}=\mathscr{E} V_{t}=\mathscr{E} L V-\mathscr{E} A V=B-\mathscr{E} A V$. Thus,

$$
\operatorname{Re}\left(R V_{t}, V\right) \leq-\operatorname{Re}(R \mathscr{E} A V, V)+c\|V(t)\|^{2}+\|B(t)\|\|V(t)\| .
$$

Therefore

$$
\frac{d}{d t} \frac{1}{2}(R(t) V(t), V(t)) \leq-\operatorname{Re}(R \mathscr{E} A V, V)+c\|V(t)\|^{2}+\|B(t)\|\|V(t)\| .
$$

Then (3.7) for $s=0$ follows upon integration if we can prove that

$$
|\operatorname{Re}(R \mathscr{E} A V(t), V(t))| \leq c\|V(t)\|^{2} .
$$

In case $L$ is symmetric hyperbolic one can take $R=I$ and the proof of (3.8) is elementary. Simply use the fact that $\mathscr{E}$ is selfadjoint and $\mathscr{E} V=V$ to write

$$
\operatorname{Re}(\mathscr{E} A V, V)=\operatorname{Re}(A V, \mathscr{E} V)=\left(\left(A+A^{*}\right) V, V\right) / 2 \leq c\|V(t)\|^{2},
$$

where the last estimate follows since $A+A^{*}$ is a differential operator of order zero.

In the general case, write $V=V_{1}+V_{2}$ with

$$
V_{1}=\left((V)_{1}, 0, \ldots, 0\right) \text { and } V_{2}=(0,[V]) \text {. }
$$

Then

$$
(R \mathscr{E} A V, V)=\sum\left(R \mathscr{E} A V_{i}, V_{j}\right)=\sum\left(R A V_{i}, V_{j}\right)+\sum\left(R(\mathscr{E}-I) A V_{i}, V_{j}\right) .
$$

The first sum on the right is equal to $(R A V, V)$ which has real-part bounded by $\|V(t)\|^{2}$ since $R A+(R A)^{*}$ is bounded.

Since $V_{2}$ does not depend on $\theta$, the summands in the last term vanish if $i$ is equal to 2 , since then $(\mathscr{E}-I) A V_{i}$ vanishes. Similarly, if $j=2$ write $\left(R(\mathscr{E}-I) A V_{i}, V_{j}\right)=\left(A V_{i},(\mathscr{E}-I) R V_{j}\right)$ and the right-hand member in the scalar product vanishes.

The one remaining term is interesting. Write

$$
\left(R \mathscr{E} A V_{1}, V_{1}\right)=\left(R A V_{1}, V_{1}\right)+\left(R(I-\mathscr{E}) A V_{1}, V_{1}\right) .
$$


The real part of the first term on the right is estimated by $c\left\|V_{1}(t)\right\|^{2}$ since $\operatorname{Re}(R A)$ is bounded. Express the second term as

$$
I-\mathscr{E}=\operatorname{diag}(I-E, I-E, \ldots, I-E)+\operatorname{diag}(E-I, 0, \ldots, 0) .
$$

The contribution of the first term on the right of (3.10) to the second term on the right of (3.9) is equal to $\left(R \operatorname{diag}(I-E) A V_{1}, V_{1}\right)$. The diagonal operator is a projection which acts only on the $\theta$ variables so it commutes with $R$ and $A$. This yields the equivalent expression $\left(R A(I-E) V_{1},(I-E) V_{1}\right)$. Therefore,

$$
\left|\operatorname{Re}\left(R \operatorname{diag}(I-E) A V_{1}, V_{1}\right)\right| \leq c\left\|(I-E) V_{1}\right\|^{2},
$$

as above.

The final term is equal to

$$
\begin{aligned}
\left(R \operatorname{diag}(E-I, 0, \ldots, 0) A V_{1}, V_{1}\right) & =\left(V_{1}, R^{1,1}(x, D)(E-I) A^{1,1}(x, D) V_{1}\right) \\
& =\left((E-I) V_{1}, R^{1,1} A^{1,1}(E-I) V_{1}\right),
\end{aligned}
$$

since $E$ commutes with the scalar operators $R^{1,1}$ and $A^{1,1}$. The symbol of $R^{1,1}$ is real since $R=R^{*}$. The expression $\sum A_{j}^{1,1} \partial_{j}$ is equal to the directional derivatives along the rays on the characteristic surfaces $\varphi=$ constant [RR2]. In particular $A_{j}^{1,1}$ is real so $A_{j}^{1,1} \partial_{j}$ has imaginary symbol. Thus $R^{1,1} A^{1,1}$ has pure imaginary principal symbol and it follows that

$$
\left|\operatorname{Re}\left((E-I) V_{1}, R^{1,1} A^{1,1}(E-I) V_{1}\right)\right| \leq c\left\|(E-I) V_{1}\right\|^{2} .
$$

This completes the proof of $(3.8)$.

For general $s$ and $V \in H^{s+1}\left([0, T] \times \mathbb{R}^{n} \times S^{1}\right),(3.7)$ is proved in the same way starting with $\frac{d}{d t}\left(\left(1-\Delta_{\tilde{x}, \theta}\right)^{s} V(t), V(t)\right)$.

The uniqueness part of Proposition 3.1 follows since if $V$ satisfies the initial value problem with $\alpha=0$ and $B=0$ then solving for $V_{t}$ using the differential equation shows that $V \in C^{1}\left([0, T]: H^{s-1}\left(\mathbb{R}^{n} \times S^{1}\right)\right)$ and the energy estimate for $s-1$ proves that $V=0$. For later use, note that this uniqueness proof is valid under the weaker hypothesis that $V \in L^{\infty}\left([0, T]: H^{s}\left(\mathbb{R}^{n} \times S^{1}\right)\right)$.

To prove existence consider $V^{\delta}$ the solution of

$$
\begin{aligned}
& V_{t}^{\delta}+\mathscr{E} J^{\delta} A V^{\delta}=B \quad \text { and } \quad V^{\delta}(0)=\alpha, \\
& J^{\delta}(D) \equiv \exp \left(\delta \Delta_{x}\right) .
\end{aligned}
$$

Since $J^{\delta}$ is an infinitely smoothing approximation of the identity, the linear ordinary differential equation (3.11) has a unique solution in

$$
C^{\infty}\left([0, T]: H^{s}\left(\mathbb{R}^{n} \times S^{1}\right)\right) \text {. }
$$

Repeating the derivation of the energy estimate (3.7) shows that $V^{\delta}$ is bounded in $C^{m}\left([0, T]: H^{s-m}\left(\mathbb{R}^{n} \times S^{1}\right)\right.$ ) for $m=0,1$. Passing to a weakly convergent subsequence yields a $V$ with $\partial_{t}^{m} V \in L^{\infty}\left([0, T]: H^{s-m}\left(\mathbb{R}^{n} \times S^{1}\right)\right)$ for $m=0,1$ which solves the limiting equation $V_{t}+\mathscr{E} A V=B$. The solution so constructed satisfies estimate (3.7) inherited from the uniform estimates for $V^{\delta}$.

Since $\mathscr{E} B=B$, multiplying the equation $V_{t}+\mathscr{E} A V=B$ by $I-\mathscr{E}$ implies that $\partial_{t}((I-\mathscr{E}) V)=0$. Since $\mathscr{E} \alpha=\alpha$ it follows that $\left.(I-\mathscr{E}) V\right|_{t=0}$ vanishes and therefore $(I-\mathscr{E}) V \equiv 0$.

To prove that $V$ is continuous with values in $H^{s}\left(\mathbb{R}^{n} \times S^{1}\right)$ choose $\alpha_{\nu} \in$ $H^{s+1}\left(\mathbb{R}^{n} \times S^{1}\right)$ and $B_{\nu} \in L^{1}\left([0, T]: H^{s+1}\left(\mathbb{R}^{n} \times S^{1}\right)\right)$ both invariant under $\mathscr{E}$ 
and converging to $\alpha$ and $B$ in $H^{s}\left(\mathbb{R}^{n} \times S^{1}\right)$ and $L^{1}\left([0, T]: H^{s}\left(\mathbb{R}^{n} \times S^{1}\right)\right)$, respectively. The basic estimates shows that the solutions $V_{\nu}$ are a Cauchy sequence in $C\left([0, T]: H^{s}\right)$ and converge to $V$ in $C\left([0, T]: H^{s-1}\right)$. It follows that $V$ is continuous with values in $H^{s}$. Q.E.D.

Given the linear existence theorem above, the profile $U_{0}$ is constructed as the limit of the Picard iterates $U_{0}^{\nu}$ defined by

$$
\begin{aligned}
& (I-\mathscr{E}) U_{0}^{\nu}=0,\left.\quad \mathscr{E} U_{0}^{\nu}\right|_{t=0}=\left.U_{0}\right|_{t=0}=\text { given, } \\
& \mathscr{E}\left(L\left(x, \partial_{x}\right) U_{0}^{\nu}-f\left(x, U_{0}^{\nu-1}\right)-B_{0}\right)=0 .
\end{aligned}
$$

If $s>(n+1) / 2$ the map $W \mapsto f(x, W)$ is a locally Lipschitzean map of $H^{s}\left(\mathbb{R}^{n} \times S^{1}\right)$ to itself. This together with estimate (3.7) immediately yields convergence of the iterates on a small time interval $\left[0, T_{0}\right]$. In the next assertions $H^{\infty} \equiv \bigcap H^{s}$.

Theorem 3.2. Suppose that $s>(n+1) / 2, \alpha_{0} \in H^{s}\left(\mathbb{R}^{n} \times S^{1}\right)$,

$$
B_{0} \in L^{1}\left([0, T]: H^{s}\left(\mathbb{R}^{n} \times S^{1}\right)\right),
$$

and $\mathscr{E} \alpha=\alpha$. Then there is a $\left.\left.T_{0} \in\right] 0, T\right]$ and a unique solution $U_{0} \in$ $C\left(\left[0, T_{0}\right]: H^{s}\left(\mathbb{R}^{n} \times S^{1}\right)\right)$ of $(2.10)$, (2.11) with $U_{0}(0, \cdot)=\alpha$. If the data belong to $H^{\infty}\left(\mathbb{R}^{n} \times S^{1}\right)$ and $C^{\infty}\left(\left[0, T_{0}\right]: H^{\infty}\left(\mathbb{R}^{n} \times S^{1}\right)\right)$ respectively, then the solution $U_{0}$ belongs to $C^{\infty}\left([0, T]: H^{\infty}\left(\mathbb{R}^{n} \times S^{1}\right)\right)$.

Once $U_{0}$ is determined on $\left[0, T_{0}\right]$ the higher order profiles exist on the same time interval by solving a linear equation.

Theorem 3.3. Suppose that $s>(n+1) / 2, \alpha_{k} \in H^{s}\left(\mathbb{R}^{n} \times S^{1}\right)$,

$$
B_{k} \in L^{1}\left([0, T]: H^{s}\left(\mathbb{R}^{n} \times S^{1}\right)\right),
$$

$\mathscr{E} \alpha_{k}=\alpha_{k}$, for $1 \leq k \in \mathbb{N}$ and $U_{0} \in C\left(\left[0, T_{0}\right]: H^{s}\left(\mathbb{R}^{n} \times S^{1}\right)\right)$ solves (2.10), (2.11). Then for each $k \geq 1$ there is $U_{k} \in C\left(\left[0, T_{0}\right]: H^{s}\left(\mathbb{R}^{n} \times S^{1}\right)\right)$ a unique solution of (2.12), (2.13) with $\mathscr{E} U_{k}(0, \cdot)=\alpha_{k}$. If for $j \leq k$ the data belong to $H^{\infty}\left(\mathbb{R}^{n} \times S^{1}\right)$ and $C^{\infty}\left(\left[0, T_{0}\right]: H^{\infty}\right)$ respectively, then $U_{j} \in C^{\infty}\left(\left[0, T_{0}\right]: H^{\infty}\right)$ for $j \leq k$.

Remarks. 1. The hypothesis of strict hyperbolicity is not really necessary. What is needed is that the operator $L$ be symmetrizable in the adapted coordinates and that the level surfaces of $\varphi$ be simply characteristic. Even the latter hypothesis can be weakened. It suffices that the algebraic and geometric multiplicity of $\operatorname{ker}\left(L_{1}(x, d \varphi(x))\right)$ are equal and independent of $x$ near $\underline{x}$.

2. The regularity of the profiles $U_{k}$ with respect to $x$ and $\theta$ have different interpretations. Regularity in $x$ measures the smoothness of the $x$ dependence. Regularity in $\theta$ describes how rapidly the Fourier coefficients decay to zero which dictates the distribution of energy over the high frequency harmonics. It is natural to consider spaces of mixed regularity $H^{s, \rho}\left(\mathbb{R}^{n} \times S^{1}\right)$ defired by the norms

$$
\|W(x, \theta)\|_{H^{s, \rho}}^{2} \equiv \sum_{m} \int|\widehat{W}(\xi, m)|^{2}\left(1+|\xi|^{2}\right)^{s}\left(1+|m|^{2}\right)^{\rho} d \xi .
$$


The above theorems have natural $H^{s, \rho}$ versions provided $s>n / 2$ and $\rho>$ $1 / 2$. Such refinements enter in sum laws for interactions as in [JR2].

\section{Rigorous ASYMPTOTICS. I: CONTINUATION PROBLEMS}

Combining the results of the previous two sections with the theory of stratified solutions developed in [RR3], it is a simple matter to show that the formal solutions

$$
\sum \varepsilon^{k} U_{k}(x, \varphi(x) / \varepsilon),
$$

are asymptotic to exact solutions. Toward that end, suppose that

$$
U_{k} \in H^{\infty}\left(I \times \mathbb{R}^{n}\right) \quad \text { satisfies (2.1), (2.2), and (2.3), } \quad \forall k \in \mathbb{N},
$$

where $I \subset \mathbb{R}$ is a compact interval. Theorems 3.2 and 3.3 allow one to extend the $U_{k}$ to solutions on a larger compact interval $I^{\prime}$ with $I \subset \operatorname{Int}\left(I^{\prime}\right)$.

Suppose that the normalizations (3.1), (3.2), and (3.3) are satisfied. The spaces $H_{\partial^{\prime}}^{s}$ of stratified distributions are defined as follows. Let $\Omega \equiv I \times \mathbb{R}^{n}$ (respectively $\Omega^{\prime} \equiv I^{\prime} \times \mathbb{R}^{n}$ ) and

$$
\partial^{\prime} \equiv\left(\partial_{0}, \partial_{1}, \ldots, \partial_{n-1}\right),
$$

be the derivatives parallel to the foliation by the level surfaces of the phase $\varphi(x)=x_{n}$. Then

$$
H_{\partial^{\prime}}^{s}(\Omega) \equiv\left\{u \in L^{2}(\Omega):|\alpha| \leq s \Rightarrow\left(\partial^{\prime}\right)^{\alpha} u \in L^{2}(\Omega)\right\} .
$$

Given a formal series as in (4.1) there is a family $u^{\varepsilon}(x)=u(x, \varepsilon)$ which has the series as asymptotic expansion. To prove that, use a variant of Borel's Theorem to construct a $U(x, \theta, \varepsilon)$ in $H^{\infty}\left(\Omega^{\prime} \times S^{1} \times[0,1]\right)$ such that $\left.\partial_{\varepsilon}^{k} U\right|_{\varepsilon=0}=U_{k}$ and moreover,

$$
U(x, \theta, \varepsilon) \sim \sum \varepsilon^{k} U_{k}(x, \theta),
$$

in the sense that for all $M$ and $s$

$$
U(x, \theta, \varepsilon)-\sum \varepsilon^{k} U_{k}(x, \theta)=O\left(\varepsilon^{M+1}\right) \quad \text { in } H^{s}\left(\Omega^{\prime} \times S^{1}\right) .
$$

Defining

$$
u(x, \varepsilon) \equiv U(x, \varphi(x) / \varepsilon, \varepsilon),
$$

one has

$$
u(x, \varepsilon)-\sum^{M} \varepsilon^{k} U_{k}(x, \varphi(x) / \varepsilon)=O\left(\varepsilon^{M+1}\right) \quad \text { in } H_{\partial^{\prime}}^{s}\left(\Omega^{\prime}\right) \quad \forall s, M .
$$

The key remark is that the errors are estimated in the stratified space $H_{\partial^{\prime}}^{s}$. In the same way, one can construct $b(x, \varepsilon)$ with $\sum \varepsilon^{k} B_{k}(x, \varphi(x) / \varepsilon)$ the asymptotic expansion in $H_{\partial^{\prime}}^{\infty}(\Omega)$.

The construction of the formal solution guarantees that

$$
L u(x, \varepsilon)-f(x, u(x, \varepsilon))-b(x, \varepsilon)=r(x, \varepsilon),
$$

with

$$
r(x, \varepsilon)=O\left(\varepsilon^{M}\right) \quad \text { in } H_{\partial^{\prime}}^{s}\left(\Omega^{\prime}\right) \text { for all } s, M .
$$


Thus the formal solution is asymptotic to a family of functions $u(x, \varepsilon)$ which are nearly solutions in the sense that their residuals $r(x, \varepsilon)$ vanish faster than any power of $\varepsilon$ as $\varepsilon$ tends to zero.

Next we construct a family of exact solutions which is asymptotic to $u(x, \varepsilon)$. This is a straightforward application of the results of [RR3]. Denote by $a$ (resp. $a^{\prime}<a$ ) the left-hand endpoint of $I$ (resp. $I^{\prime}$ ). Choose a smooth function $\chi(t)$ such that $\chi$ vanishes for $t \geq a$ and $\chi=1$ for $t \leq\left(a+a^{\prime}\right) / 2$. Define $u^{\varepsilon}$ to be the solution of the following continuation problem.

$$
\begin{gathered}
L u^{\varepsilon}+f\left(x, u^{\varepsilon}(x)\right)-b(x, \varepsilon)=\chi(t) r(x, \varepsilon), \\
u^{\varepsilon}=u(x, \varepsilon) \text { for } a^{\prime} \leq t \leq\left(a+a^{\prime}\right) / 2 .
\end{gathered}
$$

The data $\chi r(x, \varepsilon)$ for $u^{\varepsilon}$ and $r(x, \varepsilon)$ for $u(x, \varepsilon)$ differ by $O\left(\varepsilon^{M}\right)$ in $\left(L^{\infty} \cap H_{\partial^{\prime}}^{s}\right)\left(\Omega^{\prime}\right)$ for all $s$ and $M$ and the solutions $u(x, \varepsilon)$ are bounded in $\left(L^{\infty} \cap H_{\partial^{\prime}}^{s}\right)\left(\Omega^{\prime}\right)$. In [RR3] $\left(L^{\infty} \cap H_{\partial^{\prime}}^{s}\right)\left(\Omega^{\prime}\right)$ well posedness is demonstrated. It follows that $u^{\varepsilon}$ exists on all of $\Omega^{\prime}$ and

$$
u^{\varepsilon}-u(x, \varepsilon)=O\left(\varepsilon^{M}\right) \quad \text { in }\left(L^{\infty} \cap H_{\partial^{\prime}}^{s}\right)\left(\Omega^{\prime}\right)
$$

for all $s$ and $M$. Summarizing we have the following result.

Theorem 4.1. Suppose that (4.1) is a formal asymptotic solution, that is the profiles satisfy (4.2). Then there is a family $u^{\varepsilon}$ of functions depending smoothly on $\varepsilon$ with values in $L^{\infty}(\Omega) \cap H_{\partial^{\prime}}^{\infty}(\Omega)$ and satisfying.

$$
\begin{gathered}
L u^{\varepsilon}+f\left(x, u^{\varepsilon}(x)\right)-b(x, \varepsilon)=0 \quad \text { in } I \times \mathbb{R}^{n}, \quad \text { and } \\
u^{\varepsilon}-\sum_{k=0}^{M} \varepsilon^{k} U_{k}(x, \varphi(x) / \varepsilon)=O\left(\varepsilon^{M+1}\right) \quad \text { in } L^{\infty}(\Omega) \cap H_{\partial^{\prime}}^{s}(\Omega) \quad \forall s, M .
\end{gathered}
$$

This theorem shows that if $U_{0}(x, \theta)$ is a solution of the integro-differential system $(2.10)-(2.11)$ then $U_{0}(x, \varphi(x) / \varepsilon)$ is the leading term of the asymptotic expansion of an exact solution.

\section{The SEMilinear CAUChy PROBlem For STRATIFIED SOlutions}

In the last section it was shown that there exist exact solutions asymptotic to the formal solutions. A more natural assertion would be that given a formal solution, exact solutions whose data are asymptotic to the series $\sum \varepsilon^{k} b_{k}(x, \varphi / \varepsilon)$ and $\sum \varepsilon^{k} \gamma_{k}(\tilde{x}, \varphi / \varepsilon)$ are asymptotic to the formal solutions. This is the content of Theorem 6.1. Theorem 3.3 shows that for arbitrary $b_{k}$ and projections $\mathscr{E} \gamma_{k}$, such formal solutions exist.

For the proof it is necessary to extend the bounded stratified theory of [RR3] in order to treat initial value problems. The modifications involve finding strengthened forms of compatibility conditions which are propagated by the evolution of $L$ and are preserved under nonlinear maps. The end result is improved control of the regularity of solutions in directions transverse to the foliation by the level curves of $\varphi$.

Supose that (3.1), (3.2), (3.3) are satisfied. Recall the definition of the stratified distributions.

$$
\partial^{\prime} \equiv\left(\partial_{0}, \partial_{1}, \ldots, \partial_{n-1}\right)
$$




$$
H_{\partial^{\prime}}^{s}\left(\Omega_{T}\right) \equiv\left\{u \in L^{2}\left(\Omega_{T}\right):|\alpha| \leq s \Rightarrow\left(\partial^{\prime}\right)^{\alpha} u \in L^{2}\left(\Omega_{T}\right)\right\},
$$

where $\Omega_{T} \equiv[0, T] \times \mathbb{R}^{n}$. Similarly

$$
\partial^{\prime \prime} \equiv\left(\partial_{1}, \partial_{2}, \ldots, \partial_{n-1}\right),
$$

are the spatial derivatives in $\partial^{\prime}$.

$$
H_{\partial^{\prime \prime}}^{s} \equiv\left\{g \in L^{2}\left(\mathbb{R}^{n}\right):|\alpha| \leq s \Rightarrow\left(\partial^{\prime \prime}\right)^{\alpha} u \in L^{2}\left(\mathbb{R}^{n}\right)\right\}=L^{2}\left(x_{n}: H^{s}\left(\mathbb{R}^{n-1}\right)\right) .
$$

Even in the linear case, the initial value problem with stratified data, that is $g \in H_{\partial^{\prime \prime}}^{s}$, does not usually have a stratified solution. One expects $N$ waves with $N$ different stratifications, one for each family of characteristic surfaces passing through the initial foliation $\left\{t=0\right.$ and $x_{n}=$ constant $\}$. In the nonlinear case these waves can interact to generate, by resonance, other waves and in general the situation will be impossibly complicated [JR4]. To insure that only one wave train emerges, compatibility conditions at $t=0$ must be imposed on the data.

Consider first the Cauchy problem

$$
L u=b \in H_{\partial^{\prime}}^{s}, \quad s \geq 1, \quad \text { and } \quad u(0, \cdot)=g_{0} \in L^{2}\left(\mathbb{R}^{n}\right) .
$$

The solution belongs to $C\left([0, T]: L^{2}\left(\mathbb{R}^{n}\right)\right) \subset C\left([0, T]: \mathscr{D}^{\prime}\left(\mathbb{R}^{n}\right)\right)$. Note that $b \in C^{s-1}\left([0, T]: \mathscr{D}^{\prime}\right)$ so solving for $u_{t}$ using the equation one finds that $u \in$ $C^{1}\left([0, T]: \mathscr{D}^{\prime}\right)$. Continuing in this fashion one shows that $u \in C^{s}\left([0, T]: \mathscr{D}^{\prime}\right)$ and that the derivatives $\left.g_{j} \equiv \partial_{t}^{j} u\right|_{t=0}$ for $0 \leq j \leq s$ are given by equations of the form

$$
g_{j}=\left.\partial_{t}^{j-1} b\right|_{t=0}+\sum_{m \leq j-1} c_{m, j}\left(x, \partial_{x}\right) g_{m},
$$

with $c_{m, j}$ of order $m-j$. A commutation argument [RR2,3] yields the following linear stratified existence theorem.

Proposition 5.1. Suppose $s \in \mathbb{N}, b \in H_{\partial^{\prime}}^{s}, g_{0} \in L^{2}\left(\mathbb{R}^{n}\right)$, and $u \in L^{2}\left(\Omega_{T}\right)$ is the solution of (5.5). If $g_{j}$, defined by the formulas (5.6), satisfy

$$
\mathscr{C}_{s}: g_{j} \in H_{\partial^{\prime \prime}}^{s-j} \text { for } j=0,1, \ldots, s,
$$

then $u \in H_{\partial^{\prime}}^{s}$. In fact $\partial_{t}^{j} u \in C\left([0, T]: H_{\partial^{\prime \prime}}^{s-j}\right)$ for $j \leq s$ and

$$
|u(t)|_{s, \partial^{\prime}} \leq c\left(|u(0)|_{s, \partial^{\prime}}+\int_{0}^{t}|b(\sigma)|_{s, \partial^{\prime}} d \sigma\right),
$$

where $c=c(s, T, L, \varphi)$ and

$$
|u(t)|_{s, \partial^{\prime}} \equiv \sum_{|\alpha| \leq s}\left\|\left(\partial^{\prime}\right)^{\alpha} u(t)\right\|_{L^{2}\left(\mathbb{R}^{n}\right)} .
$$

Elements of the space $H_{\partial}^{s}$, need be no better than $L^{2}$ in $x_{n}$. In particular they do not form an algebra no matter how large is $s$. In order to treat nonlinear problems we seek additional regularity in $x_{n}$ which is propagated by the equation and preserved under nonlinear operations. 
Proposition 5.2. Suppose in addition to the hypotheses $\mathscr{C}_{s}$ of Proposition 5.1, that

$$
\mathscr{C}_{s-1}^{\prime}\left(\begin{array}{l}
(5.9) \partial_{t}^{j} b \in L^{1}\left([0, T]: C\left(x_{n}: H_{\partial^{\prime \prime}}^{s-j-3 / 2}\left(\mathbb{R}_{x^{\prime \prime}}^{n-1}\right)\right)\right) \quad \text { for } 0 \leq j \leq s-1, \\
(5.10) g_{j} \in C\left(x_{n}: H_{\partial^{\prime \prime}}^{s-j-3 / 2}\left(\mathbb{R}_{x^{\prime \prime}}^{n-1}\right)\right) \quad \text { for } j=0,1, \ldots, s-1 .
\end{array}\right.
$$

Then the solution $u$ to (5.5) satisfies:

$$
u \in \bigcap_{j=0}^{s-1} C^{j}\left([0, T]: C\left(x_{n}: H_{\partial^{\prime \prime}}^{s-j-3 / 2}\left(\mathbb{R}_{x^{\prime \prime}}^{n-1}\right)\right)\right) .
$$

Remark. There are estimates corresponding to the regularity of $u$. To simplify matters consider the case where $b$ and $g_{j}$ are bounded continuous functions of $x_{n}$ so that the data naturally belong to Banach spaces denoted $\underline{C}\left(x_{n}: \cdot\right)$ the symbol $\underline{C}$ indicating the set of bounded continuous functions. The norm is then $\sup \{\|u(t)\|: 0 \leq t \leq T\}$ where

$$
\|u(t)\| \equiv|u(t)|_{s, \partial^{\prime}}+\sup _{j, x_{n}}\left\|u\left(t, x_{n}\right)\right\|_{H^{s-j-3 / 2\left(\mathbb{R}_{x^{\prime \prime}}^{n-1}\right)}} .
$$

A quantitative version of the proposition is

$$
\|u(t)\| \leq c\left(\|u(0)\|+\int_{0}^{t} \sum\left\|\partial_{t}^{j} b(\sigma)\right\|_{\underline{C}\left(x_{n}: H^{\left.s-j-3 / 2\left(\mathbb{R}^{n-1}\right)\right)}\right.} d \sigma\right) .
$$

Proof. First we show that for the last $N-1$ components of $u$, denoted [ $u$ ], the desired regularity follows from conormal regularity and the differential equation. Here one does not need $\mathscr{C}_{s-1}^{\prime} \equiv(5.9)-(5.10)$.

The differential equation can be solved for $\partial_{n}[u]$ by expressing the equation as a linear combination of $\partial_{t}[u], \partial^{\prime \prime} u$, and $b$. Proposition 5.1 implies that

$$
u \in \bigcap_{j=0}^{s} C^{j}\left([0, T]: L^{2}\left(x_{n}: H_{\partial^{\prime \prime}}^{s-j}\left(\mathbb{R}_{x^{\prime \prime}}^{n-1}\right)\right)\right) .
$$

Therefore

$$
\left\{\partial_{t} u, \partial^{\prime \prime} u\right\} \subset \bigcap_{j=0}^{s-1} C^{j}\left([0, T]: L^{2}\left(x_{n}: H_{\partial^{\prime \prime}}^{s-j-1}\left(\mathbb{R}_{x^{\prime \prime}}^{n-1}\right)\right)\right) .
$$

Since $b \in H_{\partial^{\prime}}^{s}$ the standard trace theorem yields

$$
b \in \bigcap_{j=0}^{s-1} C^{j}\left([0, T]: L^{2}\left(x_{n}: H_{\partial^{\prime \prime}}^{s-j-1 / 2}\left(\mathbb{R}_{x^{\prime \prime}}^{n-1}\right)\right)\right) .
$$

This is better than the regularity of the derivatives of $u$ above so $\partial_{n}[u]$ has the regularity of the latter derivatives, namely

$$
\partial_{n}[u] \in \bigcap_{j=0}^{s-1} C^{j}\left([0, T]: L^{2}\left(x_{n}: H_{\partial^{\prime \prime}}^{s-j-1}\left(\mathbb{R}_{\chi^{\prime \prime}}^{n-1}\right)\right)\right) .
$$

This together with (5.12) implies that

$$
[u] \in \bigcap_{j=0}^{s-1} C^{j}\left([0, T]: \underline{C}\left(x_{n}: H_{\partial^{\prime \prime}}^{s-j-1 / 2}\left(\mathbb{R}_{x^{\prime \prime}}^{n-1}\right)\right)\right),
$$


which is one $\partial^{\prime \prime}$ derivative stronger than the proposition requires.

Next the hypotheses (5.9), (5.10) and a transport equation are used to derive the desired regularity of the first component of $u$. Recall that

$$
\sum A_{j}^{1,1} \partial_{j}=\partial_{t}+\sum_{1 \leq j} A_{j}^{1,1} \partial_{j}=\partial_{t}+\sum_{1 \leq j \leq n-1} A_{j}^{1,1} \partial_{j} \equiv X,
$$

is a vector field tangent to the rays which sweep out the leaves of the foliation by level surfaces of $\varphi=x_{n}$. There is not $x_{n}$ component of $X$ thanks to the block form (2.6) of $A_{n}$. The first component of the equation $L u=b$ implies that

$$
X u_{1}=\text { combination of } \partial^{\prime \prime}[u] \text { and } b_{1} \text {. }
$$

Differentiating (5.13) yields

$$
\partial^{\prime \prime}[u] \in \bigcap_{j=0}^{s-1} C^{j}\left([0, T]: \underline{C}\left(x_{n}: H_{\partial^{\prime \prime}}^{s-j-3 / 2}\left(\mathbb{R}_{x^{\prime \prime}}^{n-1}\right)\right)\right) .
$$

This and the hypothesis (5.9) show that

$$
\left\{\partial^{\prime \prime}[u], b_{1}\right\} \subset \underline{C}\left(x_{n}: H_{\partial^{\prime \prime}}^{s-3 / 2}\left([0, T] \times \mathbb{R}_{x^{\prime \prime}}^{n-1}\right)\right) .
$$

Thus for $u_{1}$ one has an initial value problem

$$
X u_{1} \in \underline{C}\left(x_{n}: H_{\partial^{\prime \prime}}^{s-3 / 2}\left([0, T] \times \mathbb{R}_{x^{\prime \prime}}^{n-1}\right)\right),\left.\quad u_{1}\right|_{t=0} \in \underline{C}\left(x_{n}: H_{\partial^{\prime \prime}}^{s-3 / 2}\left(\mathbb{R}_{x^{\prime \prime}}^{n-1}\right)\right) .
$$

The right-hand side of the transport equation belongs to

$$
L^{1}\left([0, T]: \underline{C}\left(x_{n}: H_{\partial^{\prime \prime}}^{s-3 / 2}\left(\mathbb{R}_{x^{\prime \prime}}^{n-1}\right)\right)\right) .
$$

Integrating yields $u \in C\left([0, T]: \underline{C}\left(x_{n}: H_{\partial^{\prime \prime}}^{s-3 / 2}\left(\mathbb{R}_{x^{\prime \prime}}^{n-1}\right)\right)\right)$, the variable $x_{n}$ being viewed as a parameter. Similarly, differentiating the transport equation with respect to time and using (5.10) for the initial condition yields

$$
\partial_{t}^{j} u_{1} \in C\left([0, T]: H_{\partial^{\prime \prime}}^{s-j-3 / 2}\left(\mathbb{R}_{x^{\prime \prime}}^{n-1}\right)\right) \text { for } j=0, \ldots, s-1 \text {. Q.E.D. }
$$

Remark. If $s-3 / 2>(n-1) / 2$ the solutions $u$ are bounded and continuous. This provides a good $L^{-1}$ invariant algebra in which to study semilinear problems.

For nonlinear problems it is important that our solutions be bounded so that $f(x, u)$ makes sense. In the next computation $\left.\Omega \equiv \Omega_{T} \equiv\right] 0, T\left[\times \mathbb{R}^{n}\right.$. If $u \in H_{\partial^{\prime}}^{s}(\Omega) \cap L^{\infty}(\Omega)$, the inequalities of Gagliardo-Nirenberg imply that

$$
|\alpha| \leq s \Rightarrow\left(\partial^{\prime}\right)^{\alpha} u \in L^{2 s /|\alpha|}(\Omega) .
$$

For $|\alpha|<s$, let $v \equiv\left(\partial^{\prime}\right)^{\alpha} u$ so $v \in L^{2 s /|\alpha|}$ and $v_{t} \in L^{2 s /(|\alpha|+1)}$ so $v \in$ $C\left([0, T]: \mathscr{D}^{\prime}\left(\mathbb{R}^{n}\right)\right)$. In addition, $\partial_{t}|v|^{p}=p v|v|^{p-2} v_{t} \in L^{1}(\Omega)$ for $p \equiv$ $(2 s-1) /|\alpha|$ so $\|v(t)\|_{L^{p}} \in C([0, T])$. It follows that $v \in C\left([0, T]: L^{p}\left(\mathbb{R}^{n}\right)\right)$ so

$$
|\alpha|<s \Rightarrow\left(\partial^{\prime}\right)^{\alpha} u \in C\left([0, T]: L^{(2 s-1) /|\alpha|}\left(\mathbb{R}^{n}\right)\right) .
$$

In particular, a necessary condition on $\left\{g_{j}\right\}$ so that they be the traces of an element of $H_{\partial^{\prime}}^{s} \cap L^{\infty}$ is

$$
g_{j} \in H_{\partial^{\prime \prime}}^{s-j}\left(\mathbb{R}^{n}\right) \cap L^{(2 s-1) / j}\left(\mathbb{R}^{n}\right) \text { for } j=0,1, \ldots, s .
$$

We will construct solutions with greater regularity than $H_{\partial}^{s} \cap L^{\infty}$ and for that there are correspondingly stronger conditions on $g_{j}$. A lifting, which is needed to find a first approximation to initiate Picard iteration, is provided by the next lemma. 
Lemma 5.3. Suppose that $s, \sigma \in \mathbb{R}$ and (5.17)

$g_{j} \in H_{\partial^{\prime \prime}}^{s-j}\left(\mathbb{R}^{n}\right), \quad j \leq s, \quad$ and $\quad g_{j} \in \underline{C}\left(x_{n}: H^{\sigma-j}\left(\mathbb{R}_{x^{\prime \prime}}^{n-1}\right)\right), \quad j \leq s-1$.

Then there is a

$$
u \in \bigcap_{j=0}^{s} C^{j}\left(\mathbb{R}_{t}: H_{\partial^{\prime \prime}}^{s-j}\left(\mathbb{R}^{n}\right)\right) \cap \bigcap_{j=0}^{s-1} C^{j}\left(\mathbb{R}_{t}: \underline{C}\left(x_{n}: H^{\sigma-j}\left(\mathbb{R}_{x^{\prime \prime}}^{n-1}\right)\right)\right),
$$

such that $\left.\partial_{t}^{j} u\right|_{t=0}=g_{j}$ for $j=0,1, \ldots, s$. The lifting $u$ can be chosen so that the map $\left\{g_{j}\right\} \mapsto u$ maps bounded sets to bounded sets.

Remark. If $s>n / 2$ then $u \in H_{\partial^{\prime}}^{s} \cap L^{\infty}$.

Proof of the lemma. Choose $\varphi \in C_{0}^{\infty}\left(\mathbb{R}^{n-1}\right)$ such that $\varphi\left(\xi^{\prime \prime}\right)=1$ if $\left|\xi^{\prime \prime}\right| \leq 1$. The lifting $u$ is defined by

$$
\hat{u}\left(t, x_{n}, \xi^{\prime \prime}\right) \equiv \varphi\left(t \xi^{\prime \prime}\right) \sum_{j=0}^{s} \hat{g}_{j}\left(x_{n}, \xi^{\prime \prime}\right) t^{j} / j !
$$

where - denotes partial Fourier transform with respect to $x^{\prime \prime}$. Then $u \in$ $C^{\infty}\left(\mathbb{R}_{t}: \mathscr{S}^{\prime}\left(\mathbb{R}_{x}^{n}\right)\right)$ and $\left.\partial_{t}^{j} u\right|_{t=0}=g_{j}$ thanks to the choice of $\varphi$.

To verify (5.18) calculate the derivatives

$$
\partial_{t}^{l} \hat{u}=\sum_{j=0}^{s} \sum_{m=0}^{l} \psi_{m, l, j}\left(t \xi^{\prime \prime}\right) \hat{g}_{j+l-m}\left(x_{n}, \xi^{\prime \prime}\right),
$$

where we have set $g_{j} \equiv 0$ for $j>s$ and

$$
\psi_{m, l, j}\left(t, \xi^{\prime \prime}\right) \equiv \frac{l !}{m !(l-m) !} \frac{t^{j}}{j !} \sum_{|\alpha|=m}\left(\xi^{\prime \prime}\right)^{\alpha} \partial^{\alpha} \varphi\left(t \xi^{\prime \prime}\right) .
$$

Therefore,

$$
\left\langle\xi^{\prime \prime}\right\rangle^{\tau-l} \partial_{t}^{l} \hat{u}=\sum_{j, m} \psi_{m, l, j}\left\langle\xi^{\prime \prime}\right\rangle^{j-m}\left(\left\langle\xi^{\prime \prime}\right\rangle^{\tau-j-l+m} \hat{g}_{j+l-m}\left(x_{n}, \xi^{\prime \prime}\right)\right) .
$$

For $\tau=s$ (resp. $\sigma$ ) the term in parentheses belongs to $L^{2}\left(\mathbb{R}_{x_{n}, \xi^{\prime \prime}}^{n}\right)$ (resp. $\underline{C}\left(x_{n}: L^{2}\left(\mathbb{R}_{\xi^{\prime \prime}}^{n-1}\right)\right)$. The factor in front of the parentheses is a sum of terms of the form

$$
t^{j}\left\langle\xi^{\prime \prime}\right\rangle^{j-m}\left(\xi^{\prime \prime}\right)^{\alpha} \Phi\left(t \xi^{\prime \prime}\right), \quad|\alpha|=m,
$$

where the value of $\Phi$ is clear. Since the support of the $\Phi$ term is contained in $\left|t \xi^{\prime \prime}\right| \leq c$, the expressions are uniformly bounded for $t, \xi^{\prime \prime} \in \mathbb{R} \times \mathbb{R}^{n-1}$ and the result follows. Q.E.D.

The solutions constructed below belong to

$$
C\left(t: H_{\partial^{\prime}}^{s}\left(\mathbb{R}^{n}\right) \cap \underline{C}\left(\mathbb{R}^{n}\right)\right) \cap C\left(t: L^{2}\left(\mathbb{R}^{n}\right)\right) .
$$

If $s>n / 2$, the Galiardo-Nirenberg inequalities imply that

$$
\forall|\alpha| \leq s, \quad \partial^{\alpha} u \in C\left(t: H_{\partial^{\prime}}^{s-|\alpha|}\left(\mathbb{R}^{n}\right) \cap L^{2 s /|\alpha|}\left(\mathbb{R}^{n}\right)\right) .
$$


Leibniz' rule shows that the set of such functions is invariant under the map $u \mapsto f(x, u)$. In the same way if $s>(n+2) / 2, C\left(t: \underline{C}\left(x_{n}: H_{\partial^{\prime \prime}}^{s-3 / 2}\left(\mathbb{R}^{n-1}\right)\right)\right)$ is invariant under the map $u \mapsto f(x, u)$.

The derivatives $\partial_{t}^{j} u(t)$ of solutions constructed below are continuous functions of time with values in $Y_{s-j}$ where the spaces $Y$ take account of the shift in the indices in $\mathscr{C}_{s}$ and $\mathscr{C}_{s-1}^{\prime}$ :

$$
\begin{aligned}
Y_{0} & \equiv L^{2}\left(\mathbb{R}^{n}\right), \quad \text { and } \\
Y_{s-j} & \equiv H_{\partial^{\prime \prime}}^{s-j}\left(\mathbb{R}^{n}\right) \cap \underline{C}\left(x_{n}: H^{s-j-3 / 2}\left(\mathbb{R}_{x^{\prime \prime}}^{n-1}\right)\right), \quad 0 \leq j \leq s-1 .
\end{aligned}
$$

Thus

$$
u \in B_{s} \equiv \bigcap_{j=0}^{s} C^{j}\left([0, T]: Y_{s-j}\right) .
$$

Gagliardo-Nirenberg implies that if $s>(n+2) / 2$, then

$$
\forall|\alpha| \leq s, \quad \partial^{\alpha} u \in C\left(t: L^{2 s /|\alpha|}\left(\mathbb{R}^{n}\right) \cap \underline{C}\left(x_{n}: L^{(s-3 / 2) 2 s /|\alpha|}\left(\mathbb{R}^{n-1}\right)\right)\right) .
$$

If $b \in H_{\partial^{\prime}}^{s}\left(\Omega_{T}\right)$ and $u \in B_{s}$ is a solution to $L u=f(x, u)+b,\left.u\right|_{t=0}=g_{0}$, then $f(x, u)$ belongs to $C^{s-1}\left(t: \mathscr{D}^{\prime}\left(\mathbb{R}^{n}\right)\right)$ and formula (5.6) with $b$ replaced by $b+f(u)$ together with the remark in the previous paragraph permit one to compute inductively the derivatives $\left.\partial_{t}^{j} u\right|_{t=0}=g_{j}$, for $j \leq s-1$, in terms of $g_{0}$ and $b$.

Theorem 5.4. Suppose that $\mathbb{N} \ni s>(n+3) / 2$ and $g_{0} \in H_{\partial^{\prime \prime}}^{s}\left(\mathbb{R}^{n}\right)$ satisfies

$$
\text { for } 0 \leq j \leq s, \quad g_{j} \in Y_{s-j},
$$

where $g_{j}$ are determined as above. In addition suppose that

$$
\partial_{t}^{j} b \in L^{1}\left([0, T]: Y_{s-j}\right) \text { for } j \leq s .
$$

Then there is a $\underline{T} \in] 0, T]$ and a unique

$$
u \in B_{s} \equiv \bigcap_{j=0}^{s} C^{j}\left([0, \underline{T}]: Y_{s-j}\right) \subset H_{\partial^{\prime}}^{s} \cap L^{\infty}\left(\Omega_{\underline{T}}\right),
$$

satisfying

$$
L u=f(x, u)+b \text { in }] 0, \underline{T}\left[\times \mathbb{R}^{n} \text { and } u(0, \cdot)=g_{0} .\right.
$$

The time of existence $\underline{T}$ is bounded away from zero on bounded sets of data normed in the natural way associated with the hypotheses. The map $\left\{b, g_{j}\right\} \mapsto u$ is a uniformly Lipshitzean function of the data from such bounded sets to $B_{s}$. If the data satisfy (5.21), (5.22) with $s$ replaced by $s^{\prime}>s$ then the solution belongs to $C^{j}\left([0, \underline{T}]: Y_{s^{\prime}-j}\right)$ for all $j \leq s^{\prime}$.

Proof. Let $u^{1} \in \bigcap_{j=0}^{s} C^{j}\left(\mathbb{R}_{t}: Y_{s-j}\right)$ be a lifting of the $g_{j}$ as provided in Lemma 5.3.

For $\nu \geq 2$ define $u^{\nu}$ inductively by

$$
\left.L u^{\nu}=f\left(x, u^{\nu-1}\right)+b \text { in }\right] 0, T\left[\times \mathbb{R}^{n} \text { and } u^{\nu}(0, \cdot)=g_{0}\right. \text {. }
$$


It follows inductively that

$$
\begin{aligned}
& f\left(x, u^{\nu-1}\right)+b \in \bigcap_{j=0}^{s} C^{j}\left([0, T]: Y_{s-j}\right), \quad \text { and } \\
& \left.\partial_{t}^{j} u^{\nu}\right|_{t=0}=g_{j} \text { for } j \leq s-1 .
\end{aligned}
$$

Thus the hypotheses of Lemmas 5.1 and 5.2 are satisfied and

$$
u^{\nu} \in \bigcap_{j=0}^{s} C^{j}\left([0, T]: Y_{s-j}\right)
$$

The estimates of Lemmas 5.1 and 5.2 together with estimates for $f\left(x, u^{\nu-1}\right)$ which make quantitative the invariance of $Y_{s}$ under nonlinear maps show that there is a $\underline{T}>0$ so that the $u^{\nu}$ converge in $C^{j}\left([0, \underline{T}]: Y_{s-j}\right)$ to a solution $u$ of (5.23). The dependence of $\underline{T}$ and $u$ on the data are proved in the standard manner.

Uniqueness of bounded solutions of (5.23) is classical. Q.E.D.

An important class of data $b, g$ which are compatible in the sense of (5.7), (5.21), (5.22) are the data associated with the asymptotic solutions of $\S \S 2$ and 3. Consider

$$
b^{\varepsilon}=B_{0}(x, \varphi(x) / \varepsilon)+\cdots+\varepsilon^{M} B_{M}(x, \varphi(x) / \varepsilon),
$$

and with $\tilde{x} \equiv\left(x_{1}, \ldots, x_{n}\right)$,

$$
g^{\varepsilon}=\gamma_{0}(\tilde{x}, \varphi(0, \tilde{x}) / \varepsilon)+\cdots+\varepsilon^{M} \gamma_{M}(\tilde{x}, \varphi(0, \tilde{x}) / \varepsilon) .
$$

It is clear that for any regular $B_{j}$ and $\gamma_{j}$ and any $M$ and $s$ the $b^{\varepsilon}, g^{\varepsilon}$ satisfy the compatibility conditions for each $\varepsilon>0$ fixed.

We ask under what conditions are the compatibility conditions satisfied uniformly in $\varepsilon$ as $\varepsilon$ tends to zero. That is we ask that $b^{\varepsilon}$ and $g_{j}^{\varepsilon}$ satisfy (5.21) and (5.22) with norms uniformly bounded as $\varepsilon$ tends to zero. The condition (5.22) is automatic, however the other requirement places rather strong conditions on the profiles $b_{j}$, and $\gamma_{j}$.

Recall from (2.10) and (2.12) that in order for $g^{\varepsilon}$ to be the trace at $t=0$ of a formal solution $\sum \varepsilon^{j} U_{j}(x, \varphi(x) / \varepsilon)$, the sum stopping at $j=M$, it is necessary and sufficient that

$$
(I-\mathscr{E}) \gamma_{k}=\left\{\begin{array}{l}
0 \text { for } k=0, \text { and } \\
\left.l_{k}\left(x, \gamma_{0}, \ldots, \gamma_{k-1}, B_{0}, \ldots, B_{k-1}\right)\right|_{t=0}, \quad 1 \leq k \leq M
\end{array}\right.
$$

Proposition 5.5. If $\gamma_{j}, B_{j}$ belong to $H^{\infty}\left(\mathbb{R}^{n} \times S^{1}\right)$ and $H^{\infty}\left(\Omega_{T} \times S^{1}\right)$ respectively satisfy (5.26), then for any $s \leq M+1$, the data $g^{\varepsilon}$ and $b^{\varepsilon}$ defined by (5.24), (5.25) satisfy the compatibility conditions (5.7), (5.21), (5.22) uniformly as $\varepsilon$ tends to zero.

Proof. Denote by $U_{0}, \ldots, U_{M}$ the profiles determined locally in time by the data $B_{j}, \gamma_{j}$. Theorems 3.2 and 3.3 show that such profiles exist once (5.26) is satisfied. Let

$$
u^{\varepsilon} \equiv U_{0}(x, \varphi(x) / \varepsilon)+\cdots+\varepsilon^{M} U_{M}(x, \varphi(x) / \varepsilon) .
$$

Then Proposition 2.2 shows that with $H(\varepsilon, x, \theta) \in H^{\infty}\left(\left[0,1\left[\times \Omega_{T} \times S^{1}\right)\right.\right.$,

$$
L u^{\varepsilon}=f\left(x, u^{\varepsilon}\right)+b^{\varepsilon}+\varepsilon^{M} r^{\varepsilon}, \quad r^{\varepsilon}=H(\varepsilon, x, \varphi(x) / \varepsilon) .
$$


Let $v^{\varepsilon}$ be the smooth local solution of

$$
L v^{\varepsilon}=f\left(x, v^{\varepsilon}\right)+b^{\varepsilon},\left.\quad v^{\varepsilon}\right|_{t=0}=g^{\varepsilon} .
$$

In local coordinates with $\varphi=x_{n}$, the desired conclusion is that there is a constant $c$ such that

$$
\left.\left.\left\|\partial_{t}^{j} v^{\varepsilon}(0)\right\|_{Y_{s-j}} \leq c \text { for } 0 \leq j \leq s \text { and } \varepsilon \in\right] 0,1\right] .
$$

From the definition (5.27) of $u^{\varepsilon}$ it follows that an analogous estimate with $v^{\varepsilon}$ replaced by $u^{\varepsilon}$ is valid since the derivative $\partial_{t}$ annihilates $\varphi$. Equation (5.28) then shows that the data $b^{\varepsilon}+\varepsilon^{M} r^{\varepsilon}$ and $g^{\varepsilon}$ are uniformly compatible. The strategy from here it to compare $\partial_{t}^{j} u^{\varepsilon}(0)$ and $\partial_{t}^{j} v^{\varepsilon}(0)$ for $j \leq s$.

Since $u^{\varepsilon}=v^{\varepsilon}$ at $t=0$ the equations (5.28), (5.29) yield

$$
\left.\partial_{t}\left(v^{\varepsilon}-u^{\varepsilon}\right)\right|_{t=0}=\left.\varepsilon^{M} r^{\varepsilon}\right|_{t=0} .
$$

Differentiating the equations (5.28), (5.29) with respect to $t$ and subtracting yields

$$
\left.\partial_{t}^{2}\left(v^{\varepsilon}-u^{\varepsilon}\right)\right|_{t=0}=\operatorname{Diff}_{1}\left(\partial_{t}, \partial_{x}\right)\left(f\left(v^{\varepsilon}\right)-f\left(u^{\varepsilon}\right)+\varepsilon^{M} r^{\varepsilon}\right),
$$

where $\operatorname{Diff}_{1}$ denotes a linear differential operator of degree 1 . Since $u^{\varepsilon}=v^{\varepsilon}$ at $t=0$ this simplifies to

$$
d f\left(g^{\varepsilon}\right)\left(\partial_{t} v^{\varepsilon}-\partial_{t} u^{\varepsilon}\right)+\operatorname{Diff}_{1}\left(\partial_{t}, \partial_{x}\right) \varepsilon^{M} r^{\varepsilon} .
$$

When the derivatives in the last term fall on $\varphi / \varepsilon$, the power $\varepsilon^{M}$ is reduced to $\varepsilon^{M-1}$. Thus for any $s$ and $j=0$ or $j=1$,

$$
\left.\partial_{t}^{j}\left(v^{\varepsilon}-u^{\varepsilon}\right)\right|_{t=0}=O\left(\varepsilon^{M+1-j}\right) \quad \text { in } Y_{s-j},
$$

uniformly as $\varepsilon$ tends to zero.

For general $j$ one has

$$
\left.\partial_{t}^{j}\left(v^{\varepsilon}-u^{\varepsilon}\right)\right|_{t=0}=\operatorname{Diff}_{j-1}\left(\partial_{t}, \partial_{x}\right)\left(f\left(v^{\varepsilon}\right)-f\left(u^{\varepsilon}\right)+\varepsilon^{M} r^{\varepsilon}\right) .
$$

The estimate (5.32) follows by induction for all $0 \leq j \leq M+1$. The compatibility conditions require only derivatives up to order $s$. Thus this estimate together with estimate in (5.30), with $v$ replaced by $u$, complete the proof of uniform compatibility. Q.E.D.

Example. For $M=0$, the condition in (5.26) reduces to $(1-\mathscr{E}) \gamma_{0}=0$. A direct computation yields $\partial_{t} u^{\varepsilon}=\varepsilon^{-1} A_{n} \partial_{\theta} \gamma_{0}+$ bounded terms. The condition in (5.26) for $M=0$ is seen to be equivalent to compatibility up to order $s=1+M=1$.

We believe that this equivalence is valid for all $M$.

\section{Rigorous ASYMPTOTICS. II: INITIAL VALUe PROBlems}

In this section we show that for a formal solution $\sum \varepsilon^{k} U_{k}(x, \varphi(x) / \varepsilon)$, exact solutions whose data $b(x, \varepsilon)$ and $g(x, \varepsilon)$ are asymptotic to the data of the formal solution have the formal series as asymptotic expansion. This achieves the goal set out at the end of $\S 5$.

As in $\S 4$, given profiles $U_{k} \in H^{\infty}\left(\Omega_{T} \times S^{1}\right)$ Borel's Theorem allows us to construct $U(x, \theta, \varepsilon)$ in $H^{\infty}\left(\Omega_{T} \times S^{1} \times[0,1]\right)$ and $u(x, \varepsilon) \equiv U(x, \varphi(x) / \varepsilon, \varepsilon)$ such that

$$
u(x, \varepsilon)-\sum^{M} \varepsilon^{k} U_{k}(x, \varphi(x) / \varepsilon)=O\left(\varepsilon^{M+1}\right) \quad \text { in } H_{\partial^{\prime}}^{s}\left(\Omega_{T}\right) \quad \forall s, M .
$$


In order to apply Theorem 5.4 it is important to notice that the error is small in $\underline{C}\left(x_{n}: H^{\sigma}\left(\mathbb{R}_{x^{\prime \prime}}^{n}\right)\right)$. In fact $u(x, \varepsilon)$ can be chosen so that for all $s, j, T$

$$
u(x, \varepsilon)-\sum^{M} \varepsilon^{k} U_{k}(x, \varphi(x) / \varepsilon)=O\left(\varepsilon^{M+1}\right) \quad \text { in } C^{j}\left([0, T]: Y_{s}\right)
$$

In the same way one can construct $b(x, \varepsilon)$ with the asymptotic expansion $\sum \varepsilon^{k} B_{k}(x, \varphi(x) / \varepsilon)$ in $\bigcap C^{j}\left(Y_{s}\right)$.

The construction of the formal solution guarantees that

$$
L u(x, \varepsilon)-f(x, u(x, \varepsilon))-b(x, \varepsilon)=r(x, \varepsilon),
$$

with

$$
r(x, \varepsilon)=O\left(\varepsilon^{M}\right) \text { in } C^{j}\left([0, T]: Y_{s}\right), \quad \text { for all } j, s, M .
$$

Theorem 6.1. Suppose that $U_{k}, B_{k} \in H^{\infty}\left(\Omega_{T} \times S^{1}\right)$ satisfy (2.10) to (2.13) and $u(x, \varepsilon), b(x, \varepsilon)$ are asymptotic to $\sum \varepsilon^{k} U_{k}$ and $\sum \varepsilon^{k} B_{k}$ as above. Let $u^{\varepsilon}$ be the solution of the initial value problem

$$
L u^{\varepsilon}=f\left(x, u^{\varepsilon}\right)+b(x, \varepsilon),\left.\quad u^{\varepsilon}\right|_{t=0}=\left.u(x, \varepsilon)\right|_{t=0} .
$$

Then, for $\varepsilon$ sufficiently small $u^{\varepsilon}$ exists on $\Omega_{T}$ and (6.1) holds in the sense that for all $j, s, M$,

$$
u^{\varepsilon}-\sum^{M} \varepsilon^{k} U_{k}(x, \varphi(x) / \varepsilon)=O\left(\varepsilon^{M+1}\right) \quad \text { in } C^{j}\left([0, T]: Y_{s}\right),
$$

where $Y_{s}$ is defined in (5.19).

Proof. Fix an $s>(n+3) / 2$. Proposition 5.5 shows that the data $\partial_{t}^{j} b^{\varepsilon}$ and $\left.\partial_{t}^{j} u^{\varepsilon}\right|_{t=0}$ for $j \leq s-1$ are bounded in $L^{1}\left([0, T]: Y_{s-j}\right)$ and $Y_{s-j}$ respectively.

Thus there is a $\left.\left.T_{1} \in\right] 0, T\right]$ such that the initial value problems (6.5) are solvable on $\left[0, T_{1}\right]$.

For the second step suppose that $\underline{T} \in] 0, T]$ and one has a solution $u^{\varepsilon} \in$ $C^{j}\left([0, \underline{T}]: Y_{s-j}\right)$ for $0 \leq j \leq s-1$. For $0 \leq t \leq \underline{T}$ write

$$
u^{\varepsilon}-\sum^{M} \varepsilon^{k} U_{k}(x, \varphi(x) / \varepsilon)=\left(u^{\varepsilon}-u(x, \varepsilon)\right)+\left(u(x, \varepsilon)-\sum^{M} \varepsilon^{k} U_{k}(x, \theta)\right) \text {. }
$$

The second term is estimated in (6.2). For the first use the fact that the map $b, g_{j} \mapsto u$ in Theorem 5.4 is Lipshitzean together with the equations in (6.3) to show that for $0 \leq t \leq T_{1}$

$$
\left\|\partial_{t}^{j}\left(u^{\varepsilon}-u(x, \varepsilon)\right)(t)\right\|_{Y_{s-j}} \leq c \sum_{j}\left\|\partial_{t}^{j} r(x, \varepsilon)\right\|_{L^{1}\left([0, T]: Y_{s-j}\right)}=O\left(\varepsilon^{\infty}\right) .
$$

This proves the desired asymptotic expansion up to time $\underline{T}$, in particular up to time $T_{1}$.

Choose $R$ so large that for $\varepsilon \leq 1$ and $0 \leq j \leq s-1, \partial_{t}^{j} b^{\varepsilon}$ and $\partial_{t}^{j}\left(b^{\varepsilon}+r(x, \varepsilon)\right)$ lie in the open ball of radius $R$ in $L^{1}\left([0, T]: Y_{s-j}\right)$. Increasing $R$, if necessary, one has $\partial_{t}^{j} u(x, \varepsilon)$ in the open ball of radius $R$ in $C\left([0, \underline{T}]: Y_{s-j}\right)$ for $0 \leq j \leq s-1$. $R$ can be chosen independent of $\underline{T}$.

The above estimate shows that if $u^{\varepsilon} \in C^{j}\left([0, \underline{T}]: Y_{s-j}\right)$ for $0 \leq j \leq s-1$ then there is an $\eta>0$ so that for $\varepsilon \leq \eta_{1}$ the data at time $\underline{T}$ lies in the ball of radius $R$ and the right-hand side $b^{\varepsilon}+r$ also lies in the ball of radius $R$. Thus 
there is a $\delta>0$ independent of $\underline{T}$ so that for $\varepsilon \leq \eta$ the solution extends to $[0, \min (\underline{T}+\delta, T)]$.

The first step justifies the asymptotic expansion for $0 \leq t \leq \min (\underline{T}+\delta, T)$ and shows that there is an $\eta_{2} \leq \eta_{1}$ so that the solution extends to

$$
[0, \min (\underline{T}+2 \delta, T)] \text {. }
$$

Repeating this process a finite number of times proves that there is a solution on $[0, T]$ and that the formal solution is asymtotic in the norms $Y_{s}$.

It remains to show that the solution is asymptotic in the norms $Y_{S^{\prime}}$ for all $s^{\prime}>s$. Applying the last statement of Theorem 5.4 shows that $u^{\varepsilon} \in$ $C^{j}\left([0, T]: Y_{s^{\prime}-j}\right)$ for all $j, s^{\prime}$. The second step of the present proof then justifies (6.6) with $s$ replaced by $s^{\prime}$. As $s^{\prime}$ is arbitrary, the proof is complete. Q.E.D.

A subset of the above proof proves a $Y_{s}$ asymptotic expansion when the profiles $U_{k}$ belong to $H^{s}\left(\Omega_{T} \times S^{1}\right)$ instead of $H^{\infty}$.

If $U_{0}(x, \theta)$ satisfies the integro-differential system (2.10)-(2.11) from the formal asymptotics and $\left.\gamma_{0} \equiv U_{0}\right|_{t=0}$ we can choose $\gamma_{k}$ for $k \geq 1$ so that the hypotheses of Theorem 6.1 are satisfied. In fact the equation for $U_{0}$ depends only on $B_{0}$. Given $B_{k}$ for $k \geq 0$ (for example $B_{k} \equiv 0$ ), then one need only define $\gamma_{1}, \gamma_{2}, \ldots$ inductively so that (5.26) is satisfied. Thus the initial data for $U_{0}$ is the first term of an asymptotic expansion such that the corresponding initial value problem has a solution with asymptotic expansions $U_{0}(x, \varphi / \varepsilon)+$ higher order terms.

It is important to note that this is different than saying that the solution of the initial value problem with data $B_{0}(x, \varphi / \varepsilon)$ and $\gamma_{0}(\tilde{x}, \varphi / \varepsilon)$ is equal to $U_{0}(x, \varphi / \varepsilon)+O(\varepsilon)$ in $H_{\partial^{\prime}}^{\infty}\left(\Omega_{T}\right)$. In fact the latter assertion is false. It is even false in the linear case. The reason is easy to understand. The solution of the initial value problem will contain waves $\varepsilon V\left(x, \varphi_{j} / \varepsilon\right)+$ h. o. t. where the phases $\varphi_{j}$ are the other solutions of the eikonal equation with the same initial data as $\varphi$. The data $B_{0}, \gamma_{0}$ satisfy only the first compatibility condition. For the result of the last paragraph the initial data are modified by terms of order $\varepsilon$, so as to satisfy the compatibility conditions to all orders.

The data $b(x, \varepsilon)$ and $\left.u^{\varepsilon}\right|_{t=0}$ in Theorem 6.1 converge weakly to

$$
\int B_{0}(x, \theta) d \theta \text { and }\left.\int U_{0}(x, \theta)\right|_{t=0} d \theta
$$

respectively. It is typical of strongly nonlinear problems that the weak limits of the data do not determine the weak limit of the solutions. This is true for our oscillatory solutions whose weak limit is $\int U_{0}(x, \theta) d \theta$. The very simplest examples show this.

Example. Suppose $N=1$ and so $x=\left(x_{0}, x_{1}\right) \in \mathbb{R}^{1+1}$ and consider the initial value problem

$$
\left.u_{t}+u^{2}=b^{\varepsilon}=B_{0}\left(x, x_{1} / \varepsilon\right)\right),\left.\quad u^{\varepsilon}\right|_{t=0}=0 .
$$

Equation (2.11) shows that the principal profile $U_{0}$ satisfies

$$
\partial_{t} U_{0}+U_{0}^{2}=B_{0} \text {. }
$$

Suppose that $B_{0}$ is independent of $t$. Then at $t=0$ one has

$$
U_{0}=0, \quad \partial_{t} U_{0}=B_{0}, \quad \partial_{t}^{2} U_{0}=0, \quad \partial_{t}^{3} U_{0}=-B_{0}^{2} .
$$


This yields the Taylor expansion at $t=0$,

$$
\text { weak } \lim u^{\varepsilon}=\int U_{0}(x, \theta) d \theta=\int B_{0}(x, \theta) d \theta-\left(t^{3} / 6\right) \int B_{0}^{2}(x, \theta) d \theta+\cdots .
$$

For $t>0$ this is not determined by the weak limit of $b^{\varepsilon}$.

In the case $b^{\varepsilon} \equiv 0$ and inhomogeneous initial data the formula

$$
u^{\varepsilon}(t, x)=g^{\varepsilon}\left(x_{1}\right) /\left(1-\operatorname{tg}^{\varepsilon}\left(x_{1}\right)\right),
$$

shows even more directly that the weak limit of the solution is not determined by the weak limit of the data even when $g^{\varepsilon}=G\left(x_{1}, x_{1} / \varepsilon\right)$.

A statistical approach to our oscillations would try to find a system to determine the means and other moments of the limiting solution. It is typical of such attempts that they lead to problems of closure. The equations for the moments up to a certain order require knowledge of moments of higher order. That oscillatory solutions have such closure problems is illustrated in the example. Nevertheless the system of equations for the profiles yields a closed description of their asymptotic behavior.

\section{Propagation of oscillations along rays}

Denote by $\mathscr{F}$ the foliation by level sets of the phase function $\varphi$. For bounded stratified solutions, singularities propagate along the rays for $L$ which sweep out the leaves of $\mathscr{F}$ [RR2, 3]. This section contains analogous results for oscillations. We work in the special coordinates with $\varphi=x_{n}$ and $A_{n}$ in the block form (2.6). The rays are then integral curves of the vector field $X \equiv \sum A_{j}^{1,1} \partial_{j}$.

Definition. Suppose that $U_{0} \in C\left(\Omega \times S^{1}\right)$. We say that the family $U_{0}(x, \varphi(x) / \varepsilon)$ oscillates at $\underline{x}$ if and only if $\partial_{\theta} U_{0}(\underline{x}, \cdot)$ is not identically zero. Suppose that $u^{\varepsilon}=U_{0}(x, \varphi(x) / \varepsilon)+o(\varepsilon)$ in $L_{\mathrm{loc}}^{\infty}(\Omega)$. We say that the family $u^{\varepsilon}$ oscillates at $\underline{x}$ if and only if $U_{0}$ oscillates at $\underline{x}$.

Since the last $N-1$ components of $\partial_{\theta} U_{0}$ vanish identically for formal solutions of our semilinear equations, such a family oscillates if and only if the derivative of the first component, $\partial_{\theta} U_{0}^{1}(\underline{x}, \cdot)$, is not identically zero.

Theorem 7.1. Suppose that $U_{0}(x, \theta), B_{0}(x, \theta) \in H^{\infty}\left(\Omega \times S^{1}\right)$ satisfy (2.10), (2.11), that $\Gamma$ is a connected arc of a ray in $\Omega$, and that $\partial_{\theta} B_{0}$ vanishes on $\Gamma \times S^{1}$. If $\partial_{\theta} U_{0}$ vanishes at $\underline{x} \times S^{1}$ with $\underline{x} \in \Gamma$ then $\partial_{\theta} U_{0}$ vanishes identically on $\Gamma \times S^{1}$. In particular, if $u^{\varepsilon}$ is an asymptotic solution such that $B_{0}(x, \varphi / \varepsilon)$ does not oscillate on $\Gamma$ then $u^{\varepsilon}$ oscillates at all points of $\Gamma$ or at none.

Proof. Let $v(x, \theta) \equiv \partial_{\theta} U_{0}^{1}$. Differentiation of the first component of equation (2.11) with respect to $\theta$ yields

$$
X v+c(x, \theta) v=\partial_{\theta} B_{0}^{1,1},
$$

where $c \equiv \partial f_{1}\left(x, U_{0}(x, \theta) / \partial u_{1}\right.$. By hypothesis the right-hand side vanishes on $\Gamma \times S^{1}$. Integrating the differential equation in (7.1) along $\Gamma$ shows that for any $\theta, v(\cdot, \theta)$ vanishes for all $x$ in $\Gamma$ if it vanishes at $\underline{x}$. Q.E.D.

Note that the coefficient $c$ of the ordinary differential equation for $\partial_{\theta} U_{0}$ depends on the values of $U_{0}$. Thus (7.1) is not a simple transport equation 
which determines $\partial_{\theta} U_{0}$ directly. One must solve the integro-differential system (2.10), (2.11) to determine $U_{0}$ first.

\section{TWO WAVE INTERACTION FOR TWO SPEED SYSTEMS}

The semilinear interaction of oscillatory wave trains can produce, via resonance, oscillations with an infinite number of distinct phases and thereby defy simple description [HMR, JR4]. One situation where no such resonant interaction is possible is the transverse encounter of two oscillatory wave trains for a $2 \times 2$ strictly hyperbolic system. This is the oscillatory analogue of the simplifications which occur for the interaction of conormal singularities for two speed systems [B, cas B, RR2, 3].

The description parallels the development in $\S \S 2$ through 7 and will merely be sketched. Suppose that $L$ is a $2 \times 2$ strictly hyperbolic operator with $x_{0}$ a timelike variable. Suppose that $\varphi_{i}, i=1,2$, are two real solutions of the eikonal equation such that $d \varphi_{i}$ are linearly independent. Denote by $\mathscr{F}_{i}$ the foliations defined by the level sets of $\varphi_{i}$.

If oscillations with respect to the two phases coexist on a region of space-time then terms of the form $\exp i\left(n_{1} \varphi_{1}+n_{2} \varphi_{2}\right) / \varepsilon$ will appear as source terms. If $n_{1} n_{2} \neq 0$ then $d\left(n_{1} \varphi_{1}+n_{2} \varphi_{2}\right)$ is noncharacteristic for $L$. In fact the plane spanned by the $d \varphi_{i}$ intersects the characteristic variety of $L$ in exactly the lines through $d \varphi_{i}$ and zero. This suggests that terms like $\varepsilon \exp i\left(n_{1} \varphi_{1}+n_{2} \varphi_{2}\right) / \varepsilon$ will appear in the solutions. Superposition leads to the idea of searching for asymptotic solutions of the form

$$
u^{\varepsilon} \sim U\left(x, \varphi_{1}(x) / \varepsilon, \varphi_{2}(x) / \varepsilon\right),
$$

with profile $U\left(x, \theta_{1}, \theta_{2}\right) 2 \pi$ periodic in $\theta_{1}$ and $\theta_{2}$. More generally we seek complete asymptotic expansions

$$
u^{\varepsilon} \sim \sum_{k=0}^{\infty} \varepsilon^{k} U_{k}\left(x, \varphi_{1}(x) / \varepsilon, \varphi_{2}(x) / \varepsilon\right)
$$

8.1. Formal asymptotics. Suppose that $b^{\varepsilon} \sim \sum \varepsilon^{k} B_{k}\left(x, \varphi_{1} / \varepsilon, \varphi_{2} / \varepsilon\right)$, and compute formally a series in powers of $\varepsilon$ for $L u^{\varepsilon}-f\left(x, u^{\varepsilon}\right)-b^{\varepsilon}$. Setting the coefficients of the powers of $\varepsilon$ equal to zero yields

$$
\begin{gathered}
\sum \sum A_{j}(x) \partial_{j} \varphi_{i} \partial_{\theta_{i}} U_{0}=0 \text { from } \varepsilon^{-1}, \\
L\left(x, \partial_{x}\right) U_{0}-f\left(x, U_{0}\right)-B_{0}=\sum \sum A_{j}(x) \partial_{j} \varphi_{i} \partial_{\theta_{i}} U_{0} \text { from } \varepsilon^{0} .
\end{gathered}
$$

The first equation is satisfied if both phase functions satisfy the eikonal equation and

$$
\partial_{\theta_{i}} U \in \operatorname{ker}\left(\sum_{j} A_{j} \partial_{j} \varphi_{i}\right) \quad \text { for } i=1,2 .
$$

This gives two polarization conditions on $U_{0}$.

To continue the analysis it is convenient to introduce special coordinates. Replacing $\varphi_{i}$ by $-\varphi_{i}$ if necessary we may suppose that $\varphi_{1}+\varphi_{2}$ is timelike for $L$. New local coordinates are then defined by

$$
y_{0}=\left(\varphi_{1}+\varphi_{2}\right) / 2, \quad y_{1}=\left(-\varphi_{1}+\varphi_{2}\right) / 2 \text {. }
$$


Thus without loss of generality we suppose that $\varphi_{1}=x_{0}-x_{1}$ and $\varphi_{2}=x_{0}+x_{1}$. A smooth change of basis in $\mathbb{C}^{2}$ followed by multiplication of $L$ on the left by an invertible matrix valued function [RR2] reduces $L$ to the form

$$
L=\partial_{0}+\left[\begin{array}{cc}
1 & 0 \\
0 & -1
\end{array}\right] \partial_{1}+\operatorname{Diff}_{1}\left(x, \partial^{\prime \prime}\right) .
$$

Condition (8.4) then asserts that

$$
U_{0}\left(x, \theta_{1}, \theta_{2}\right)=\left(U_{0}^{1}\left(x, \theta_{1}\right), U_{0}^{2}\left(x, \theta_{2}\right)\right) .
$$

This shows that the mixed phases $\exp i\left(n_{1} \varphi_{1}+n_{2} \varphi_{2}\right) / \varepsilon$ with $n_{1} n_{2} \neq 0$ do not appear in the principal term. This agrees with the heuristic discussion which suggested that they would appear at order $\varepsilon$.

(8.6) implies that the right hand-side of $(8.3)$ is equal to $\left(\partial_{\theta_{2}} U_{0}^{1}, \partial_{\theta_{1}} U_{0}^{2}\right)$. Introduce the averaging operator

$$
\mathscr{E} \equiv\left(E_{2}, E_{1}\right), \quad E_{j}\left(k\left(x, \theta_{1}, \theta_{2}\right)\right) \equiv \int_{0}^{2 \pi} k\left(x, \theta_{1}, \theta_{2}\right) d \theta_{j} / 2 \pi .
$$

Then the right-hand side of $(8.3)$ belongs to $\operatorname{ker}(\mathscr{E})$. The principal profile $U_{0}$ is determined by (8.6) and the integro-differential system

$$
\mathscr{E}\left(L\left(x, \partial_{x}\right) U_{0}-f\left(x, U_{0}\right)-B_{0}\right)=0 .
$$

The conditions (8.4) and (8.6) are equivalent to

$$
(I-\mathscr{E}) U_{0}=0 .
$$

For $k \geq 1$, one finds equations

$$
\begin{gathered}
(I-\mathscr{E}) U_{k}=l\left(x, U_{0}, \ldots, U_{k-1}, B_{0}, \ldots, B_{k-1}\right), \\
\mathscr{E}\left(L\left(x, \partial_{x}\right) U_{k}-D_{u} f\left(x, U_{0}\right) U_{k}-B_{k}-F_{k}\left(x, U_{0}, \ldots, U_{k-1}\right)\right)=0 .
\end{gathered}
$$

The formalism has been presented so that the equations $(8.8)$ to $(8.11)$ resemble (2.10) to (2.13). Note however that there are two angular variables $\theta_{j}$ and that the averaging operator from (8.7) is different from the averaging operator defined in (2.8), (2.10).

The formulation of analogues of Propositions 2.1 and 2.2 is left to the reader.

8.2. Solvability of the equations for the profiles. Altering $L$ and $f$, one has (3.1), (3.2), and (8.5) throughout $\mathbb{R}^{n}$.

Theorem 8.1. Theorems 3.2 and 3.3 are true if (2.10), (2.11), (2.12), and (2.13) are replaced by $(8.8),(8.9),(8.10),(8.11)$ and $S^{1}$ is replaced by $S^{1} \times S^{1}$.

Proof. As in $\S 3$, the key is to prove the energy estimate (3.7) for $s=0$ and solutions $V \in H^{1}\left(\Omega_{T} \times S^{1} \times S^{1}\right)$ to the analogue of (3.4) to (3.6). That estimate is a consequence of the analogue of (3.8).

For symmetric hyperbolic operators the proof is the same one line as in $\S 3$.

In the general case begin by writing

$$
(R \mathscr{E} A V, V)=(R A V, V)+(R(\mathscr{E}-I) A V, V) .
$$

The real part of the first term on the right is bounded by $c\|V\|^{2}$ since $R A+$ $(R A)^{*}$ is bounded. For the second term, write $V=V_{1}+V_{2}$ with $V_{1}=\left(V^{1}, 0\right)$ and $V_{2}=\left(0, V^{2}\right)$. The terms to estimate are $\operatorname{Re}\left(R(\mathscr{E}-I) A V_{i}, V_{j}\right)$. 
For $i \neq j$, the expression vanishes. Consider for example the case $i=1, j=$ 2. Then $A V_{1}$ is independent of $\theta_{2}$ so is annihilated by $E_{2}-I$. Therefore

$$
\begin{aligned}
\left(R(\mathscr{E}-I) A V_{1}, V_{2}\right) & =\left(R\left[\begin{array}{cc}
0 & 0 \\
0 & \left(E_{1}-I\right)
\end{array}\right] A V_{1}, V_{2}\right) \\
& =\left(A V_{1},\left[\begin{array}{cc}
0 & 0 \\
0 & \left(E_{1}-I\right)
\end{array}\right] R V_{2}\right) .
\end{aligned}
$$

Since $R V_{2}$ is independent of $\theta_{1}$ the last expression vanishes.

Next consider the case $i=j=1$. The case $i=j=2$ is similar.

$$
\left(R(\mathscr{E}-I) A V_{1}, V_{1}\right)=\left(R\left[\begin{array}{cc}
0 & 0 \\
0 & \left(E_{1}-I\right)
\end{array}\right] A V_{1}, V_{1}\right)=\left(R_{1,2}\left(E_{1}-I\right) A_{2,1} V^{1}, V^{1}\right) .
$$

The last expression involves only scalar-valued functions. Next use the facts that $\left(E_{1}-I\right)^{2}=E_{1}-I$ and $E_{1}$ commutes with $R_{1,2}$ and $A_{2,1}$ to show that the right-hand side is equal to $\left(\left(E_{1}-I\right) V^{1}, R_{1,2} A_{2,1}\left(E_{1}-I\right) V^{1}\right)$.

To complete the proof it suffices to show that the principal symbol of $R_{1,2} A_{2,1}$ is purely imaginary. Since the principal symbol of $R A$ is antihermitian it follows that $(R A)_{1,1}$ has purely imaginary principal symbol. Now

$$
(R A)_{1,1}=R_{1,1} A_{1,1}+R_{1,2} A_{2,1} .
$$

As in $\S 3, \partial_{0}+A_{1,1}$ is the transport operator $X$ plus lower-order terms and so has purely imaginary principal symbol. Thus $A_{1,1}$ and the first term on the right of (8.12) have purely imaginary principal symbols. It follows by subtraction that the second term on the right has purely imaginary principal symbol and the proof is complete. Q.E.D.

8.3. The striated Cauchy problem. The compatibility conditions needed for the stratified Cauchy problem disappear in the present context. Data in $H_{\partial^{\prime \prime}}^{s} \cap L^{\infty}$ lead to the solution in $H_{\partial^{\prime \prime}}^{s} \cap L^{\infty}\left(\Omega_{T}\right)$ provided that $s$ is sufficiently large. In [RR2, 3] such solutions are called striated. The explanation is that for two speed systems such data launch waves oscillating transverse to the two foliations $\mathscr{F}_{j}$ which contain all the characteristic surfaces through the initial planes $\{t=$ $0 \cap x_{1}=$ const $\}$. If the system had more than two speeds then there would exist other characteristic surfaces and compatibility conditions would be needed to avoid the corresponding waves. For $2 \times 2$ systems we have the following result from [RR3].

Theorem 8.2. Suppose that $\mathbb{N} \ni s>(n+5) / 2$,

$$
g \in H_{\partial^{\prime \prime}}^{s}\left(\mathbb{R}^{n}\right) \cap L^{\infty}\left(\mathbb{R}^{n}\right) \text { and } b \in L^{\infty}\left(\Omega_{T}\right) \cap L^{1}\left([0, T]: H_{\partial^{\prime \prime}}^{s}\left(\mathbb{R}^{n}\right)\right) .
$$

Then there is a $\underline{T} \in] 0, T]$ and a unique $u \in L^{\infty}\left(\Omega_{\underline{T}}\right) \cap C\left([0, \underline{T}]: H_{\partial^{\prime \prime}}^{s}\left(\mathbb{R}^{n}\right)\right)$ satisfying (5.23). The time of existence $\underline{T}$ is bounded away from zero on bounded sets of data normed in the natural way associated with the hypotheses. The map $b, g \mapsto u$ is a uniformly Lipshitzean map from such bounded sets to $L^{\infty}\left(\Omega_{\underline{T}}\right) \cap C\left([0, \underline{T}]: H_{\partial^{\prime \prime}}^{s}\left(\mathbb{R}^{n}\right)\right)$. If $b, g$ satisfy $(8.13)$ with an $s^{\prime}>s$ then the solution belongs to $C\left([0, \underline{T}]: H_{\partial^{\prime \prime}}^{s^{\prime}}\left(\mathbb{R}^{n}\right)\right)$.

8.4. Rigorous asymptotics. Suppose that $U_{k}$ and $B_{k}$ belong to

$$
H^{\infty}\left(\Omega_{T} \times S^{1} \times S^{1}\right) \text { for } 0 \leq k<\infty
$$


and satisfy (8.8) to (8.11) so that $\sum \varepsilon^{k} U_{k}$ is a formal asymptotic solution with source $\sum \varepsilon^{k} B_{k}$. Suppose that

$$
b(x, \varepsilon) \sim \sum \varepsilon^{k} B_{k}\left(x, \varphi_{1}(x) / \varepsilon, \varphi_{2}(x) / \varepsilon\right),
$$

in the sense that for all $M$ and $s$

$$
b(x, \varepsilon)-\sum_{j=0}^{M} \varepsilon^{k} B_{k}\left(x, \varphi_{1}(x) / \varepsilon, \varphi_{2}(x) / \varepsilon\right)=O\left(\varepsilon^{M+1}\right),
$$

in $L^{1}\left([0, T]: L^{\infty}\left(\mathbb{R}^{n}\right) \cap H_{\partial^{\prime \prime}}^{s}\left(\mathbb{R}^{n}\right)\right)$. Similarly suppose that

$$
\left.g\left(x^{\prime}, \varepsilon\right) \sim \sum \varepsilon^{k} U_{k}\left(x, \varphi_{1}(x) / \varepsilon, \varphi_{2}(x) / \varepsilon\right)\right|_{t=0},
$$

in $L^{\infty}\left(\mathbb{R}^{n}\right) \cap H_{\partial^{\prime \prime}}^{s}\left(\mathbb{R}^{n}\right)$ for all $s$.

Theorem 8.3. Let $u^{\varepsilon}$ be the solution of the initial value problem

$$
L u^{\varepsilon}=f\left(x, u^{\varepsilon}\right)+b(x, \varepsilon),\left.\quad u^{\varepsilon}\right|_{t=0}=g(x, \varepsilon) .
$$

Then for $\varepsilon$ sufficiently small $u^{\varepsilon}$ exists on $\Omega_{T}$ and

$$
u^{\varepsilon} \sim \sum \varepsilon^{k} U_{k}\left(x, \varphi_{1}(x) / \varepsilon, \varphi_{2}(x) / \varepsilon\right)
$$

in the sense that the difference between $u^{\varepsilon}$ and the sum for $k \leq M$ is $O\left(\varepsilon^{M+1}\right)$ in $C\left([0, T]: L^{\infty}\left(\mathbb{R}^{n}\right) \cap H_{\partial^{\prime \prime}}^{s}\left(\mathbb{R}^{n}\right)\right)$.

The proof and a finite expansion version analogous to Theorem 6.2 are exactly as in $\S 6$.

\subsection{Propagation of oscillations along rays.}

Definition. The family $\left.U\left(x, \varphi_{1}(x) / \varepsilon, \varphi_{2}(x) / \varepsilon\right)\right)$ oscillates transverse to the level surfaces of $\varphi_{1}$ at the point $\underline{x}$ if and only of $\partial U / \partial \theta_{1}(\underline{x}, \cdot, \cdot)$ is not identically zero. If $\left.u^{\varepsilon} \sim U\left(x, \varphi_{1}(x) / \varepsilon, \varphi_{2}(x) / \varepsilon\right)\right)+O(\varepsilon)$ in $L_{\text {loc }}^{\infty}$ then $U^{\varepsilon}$ oscillates transverse to the level surfaces of $\varphi_{1}$ at the point $\underline{x}$ if and only $U$ does.

Theorem 8.4. Suppose that $U_{0}, B_{0} \in H^{\infty}\left(\Omega \times S^{1} \times S^{1}\right)$ satisfy (8.8), (8.9) and $\Gamma \subset \Omega$ is a connected arc of a ray on a level set of $\varphi_{1}$. If $\partial B_{0} / \partial \theta_{1}$ vanishes identically on $\Gamma$ and $\partial U_{0} / \partial \theta_{1}(\underline{x}, \cdot, \cdot)$ vanishes identically at one $\underline{x} \in \Gamma$ then $\partial U_{0} / \partial \theta_{1}$ vanishes identically for all $x \in \Gamma$. In particular, if $u^{\varepsilon}$ is an asymptotic solution and $B_{0}$ does not oscillate transverse to the level sets of $\varphi_{1}$ along $\Gamma$, then $u^{\varepsilon}$ oscillates at all points of $\Gamma$ or at none.

\section{REFERENCES}

[CB] Y. Choquet-Bruhat, Ondes asymptotiques et approchées pour les systèmes d'équations aux dérivées partielles non linéaires, J. Math. Pures Appl. 48 (1969), 117-158.

[DM] R. Diperna and A. Majda, The validity of geometric optics for weak solutions of conservation laws, Comm. Math. Phys. 98 (1985), 313-347.

[E] W. E, Propagation of oscillations in the solutions of 1-d compressible fluid equations (preprint).

[G] O. Gues, These, Rennes, January 1989 (to appear).

[HK] J. Hunter and J. Keller, Weakly nonlinear high frequency waves, Comm. Pure Appl. Math. 36 (1983), 547-569. 
[HMR] J. Hunter, A. Majda, and R. Rosales, Resonantly interacting weakly nonlinear hyperbolic waves II: several space variables, Stud. Appl. Math. 75 (1986), 187-226.

[JMR] J. L. Joly, G. Metivier, and J. Rauch, Resonant one-dimensional nonlinear geometric optics (preprint).

[JR1] J. L. Joly and J. Rauch, Ondes oscillantes semi-linéaires en 1.d, Journées Equations aux Dérivées Partielles, St. Jean de Monts, Juin 1986, Publ. de l'École Polytechnique, Palaiseau.

[JR2] __ Ondes oscillantes semilinéaire à hautes fréquences, Recent Developments in Hyperbolic Equations (L. Cattabriga, F. Colombini, M. Murthy, and S. Spagnolo, eds.), Research Notes in Math., vol. 183, Pitman, New York, 1988, pp. 103-115.

[JR3] _ High frequency semilinear oscillations, Wave Motion: Theory, Modelling, and Computation (A. J. Chorin and A. J. Majda, eds.), Springer-Verlag, New York, 1987, pp. 202217.

[JR4] - Nonlinear resonance can create dense oscillations, Microlocal Analysis and Nonlinear Waves (M. Beals, R. Melrose, and J. Rauch, eds.), IMA Volumes in Math. and its Appl., Vol. 30, Springer-Verlag (to appear).

[MaRo] A. Majda and R. Rosales, Resonantly interacting weakly nonlinear hyperbolic waves I: a single space variable, Stud. Appl. Math. 71 (1984), 149-179.

[MRS] A. Majda, R. Rosales, and M. Schonbek, A canonical system of integro-differential equations in nonlinear acoustics, Stud. Appl. Math. 79 (1988), 205-262.

[MaRa] F. Massey and J. Rauch, Differentiability of solutions to hyperbolic initial-boundary value problems, Trans. Amer. Math. Soc. 189 (1974), 303-318.

[MeR] R. Melrose and N. Ritter, Interaction of nonlinear progressing waves for semilinear wave equations, Ann. of Math. 121 (1985), 187-213.

[Me] G. Metivier, The Cauchy problem for semilinear hyperbolic systems with discontinuous data, Duke Math. J. 53 (1986), 983-1011.

[RR1] J. Rauch and M. Reed, Discontinuous progressing waves for semilinear wave equations, Comm. Partial Differential Equations 10 (1985), 1033-1075.

[RR2] _ Striated solutions of semilinear two speed wave equations, Indiana Univ. Math. J. 34 (1985), 337-353.

[RR3] _ Bounded, stratified, and striated solutions of hyperbolic systems, Nonlinear Partial Differential Equations and Their Applications, Vol. IX (H. Brezis and J. L. Lions, eds.), Research Notes in Math., vol. 181, Pitman, New York, 1989.

[T] M. Taylor, Pseudodifferential operators, Princeton Univ. Press, Princeton, N.J., 1981.

Département de Mathematiques, Université de Bordeaux I, 33405 Talence Cedex, FRANCE

Department of Mathematics, University of Michigan, Ann Arbor, Michigan 48109 